\title{
A general framework for continuum damage models. I. Infinitesimal plastic damage models in stress space
}

\author{
Francisco Armero ${ }^{\mathrm{a}, *}$, Sergio Oller ${ }^{\mathrm{b}, 1}$ \\ a Structural Engineering, Mechanics and Materials, Department of Civil and Environmental Engineering, University of California, \\ 713 Davis Hall, Berkeley CA 94720, USA \\ ${ }^{\mathrm{b}}$ E.T.S. Ingenieros de Caminos, Canales y Puertos, Universitat Politècnica de Catalunya, Campus Nord, Modulo C1, 08034 Barcelona, \\ Spain
}

Received 21 August 1999; in revised form 20 January 2000

\begin{abstract}
We identify in this paper a general framework for the development of continuum damage models in their fully coupled plastic damage form. The focus of this paper is directed to the general formulation of infinitesimal models defined by yield and damage surfaces in stress space. The main feature of the proposed formulation is the direct and independent consideration of the damage mechanisms (isotropic damage, cracking, etc.) degrading the stiffness of the material, thus allowing for a complete physical characterization of these effects. This modular structure is accomplished by a kinematic decomposition of the strains in an elastic, plastic and multiple damage parts, as belonging to each activated damage mechanism. An additive decomposition in the infinitesimal range of interest is considered. Based on this decomposition, the constitutive characterization alluded to above for each damage mechanism is carried out in a complete thermodynamically consistent framework. One of the virtues of the considered framework is the fact that it includes many of the diverse damage models existing in the literature as particular cases. In this way, the developments presented herein furnish a unified framework for the formulation of continuum damage models, including isotropic damage, compliance based formulations, effective stress anisotropic models, smeared crack models and the related formulations of cracking and damage based on strong discontinuities. Besides the clear physical significance added to these existing formulations, the proposed framework also defines a very convenient context for the efficient numerical integration of the resulting models. This aspect is explored in Part II of this work, as it is the application of the framework proposed herein to the numerical simulation of porous metals. (c) 2000 Elsevier Science Ltd. All rights reserved.
\end{abstract}

Keywords: Continuum damage models; Plasticity and damage

\section{Introduction}

Damage in solids usually refers to the degradation of their elastic response on unloading. In this way, the elastic moduli of the material decrease as further damage loading occurs. Added to these effects, we can find

\footnotetext{
${ }^{*}$ Corresponding author. Fax: +1-510-643-8928.

E-mail address: armero@ce.berkeley.edu (F. Armero).

${ }^{1}$ On leave (Fall Semester 1998) at the Department of Civil and Environmental Engineering, UC Berkeley.
} 
the presence of plasticity characterized by the permanent strains in the material after full unloading. This observation has made very popular (almost universal) the formulation of plasticity models based on the kinematic decomposition of the strains in an elastic and a plastic or permanent parts. In contrast, the formulation of damage models has been based on much more diverse, and usually unrelated, frameworks.

For example, the concepts of effective stress and/or strain can be found in the literature in the development of damage models. These ideas can be traced back to the pioneering work of Kachanov (1958) in the context of isotropic damage models. The resulting models consider a scalar variable measuring the ratio between damage and intact surfaces on which the stresses act, thus defining naturally the concept of effective stress as the equivalent stress acting on the intact material. More recent references, considering also plasticity effects, thus leading to a coupled damage-plastic model, include Simo and Ju (1987a,b), Ju (1989), Lubliner et al. (1989) and Luccioni et al. (1996) among many others. Extensions of these ideas to the anisotropic case leads to the consideration of rank four tensors, defined in terms of generically called "damage tensors", relating the total and effective stresses; e.g. Cordebois and Sidoroff (1982) and

Murakami (1983), involving rank two damage tensors. The characterization of the evolution of these damage tensors is usually mathematically very complex and difficult (if not impossible) to motivate physically.

In contrast, we can find more physically motivated approaches in the modeling of damage in concrete. Following the characterization of damage as the degradation of the elastic moduli, we can find in the literature a number of models considering the fourth-order secant compliance of the material as fundamental internal variable in the formulation. The evolution of this compliance is then usually obtained in a
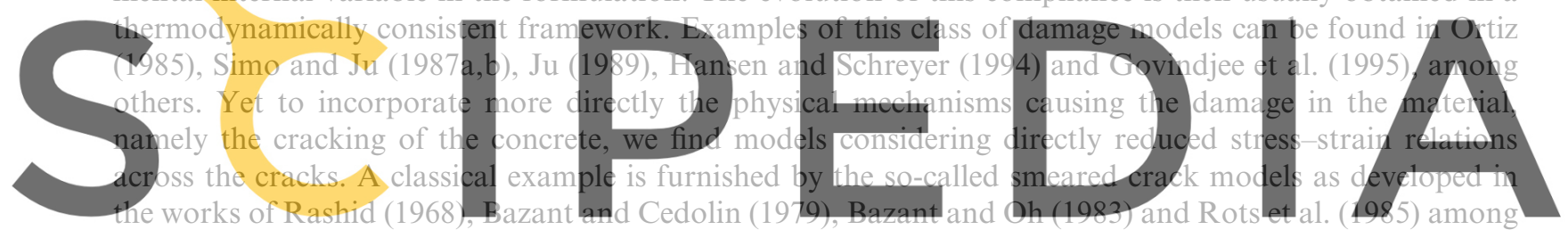

other early references. Related to these ideas, we can also quote the so-called microplane approach of

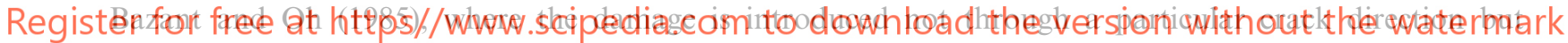
through a collection of predefined planes. Smeared crack models, however, have not been linked in the literature to the above developments in continuum damage theories. We have recently presented a formulation of these models in a completely thermodynamic framework in the context of strong discontinuities; see Armero (1997a,b), and Armero and Li (1998) for preliminary results of its extension to the finite deformation range. This context provides, in addition, the proper multi-scale treatment for the regularization of the localized failure of the material in the large-scale models of interest (Armero, 1999).

We identify in the current paper a general framework for the formulation of continuum damage models that, in particular, unifies these different treatments found in the literature, including the smeared crack models. Our attention is directed to the formulation of infinitesimal plastic damage models defined by yield and damage surfaces in stress space. The fundamental observation in the proposed framework is to base all the developments on the kinematic decomposition of the strains in an elastic, plastic and damage parts, following ideas common to elastoplastic models. With the damage strain at hand, the dissipative mechanisms causing the damage in the material can readily be modeled, independent of the elastic and other responses in the material. This allows a complete physical interpretation of the resulting equations. For example, we consider an energy potential modeling the damage stress/strain relations associated to a damage mechanism (e.g. crack stress/displacement relations), including inelastic effects like hardening/ softening cohesive laws. The presence of this damage potential identifies the recoverable nature of the damage strains. Special care is given to the identification of the different components of the stress and strain causing the damage in the material: volumetric, deviatoric, stress/strains associated to a given direction (crack), etc. 
We show the extent of the proposed framework with the consideration of the particular simple case of a quadratic damage potential, involving the evolution of a set of reduced compliances associated only to the damage mechanism. We recover then, as a particular case, the formulation presented in the seminal work of Ortiz (1985) for the purely elastic-damage case. As shown in detail herein, the framework of smeared crack models is also recovered when the identified general framework is considered in its reduced form. It is interesting to observe that even though the consideration of damage strains (sometimes in the more common form of "crack strains") can be found in these early references, their use in the formulation of continuum damage models has essentially disappeared in more recent literature. Instead, emphasis is given to the so-called "degrading strains", while maintaining the total strains decomposed only in elastic and plastic parts; representative references of these considerations are the works of Dougill (1976), Hueckel and Maier (1977), Ortiz (1987) and Yazadani and Schreyer (1988), among others. The "degrading strains" are associated to the compliance degradation of the material (i.e. $\mathbf{D} \boldsymbol{\sigma}$ for the rate of the compliance tensor D and the stress tensor $\sigma$ ). Therefore, they do not correspond to an actual "strain", in particular a recoverable strain like the damage strains, since they are only defined through a rate when damage is active. As shown in the more recent work of Carol et al. (1994), the consideration of these "degrading strains" leads to a partial unification of several of the aforementioned approaches in damage modeling, leading to a formal structure similar to elastoplastic models. We refer to this attempt as partial because models like the smeared crack approaches were not considered. In contrast, we show in this paper that the fundamental unifying assumption (in the theoretical developments and more especially at the level of the numerical integration of the resulting models, since the standard structure of return mapping algorithms common in elastoplastic
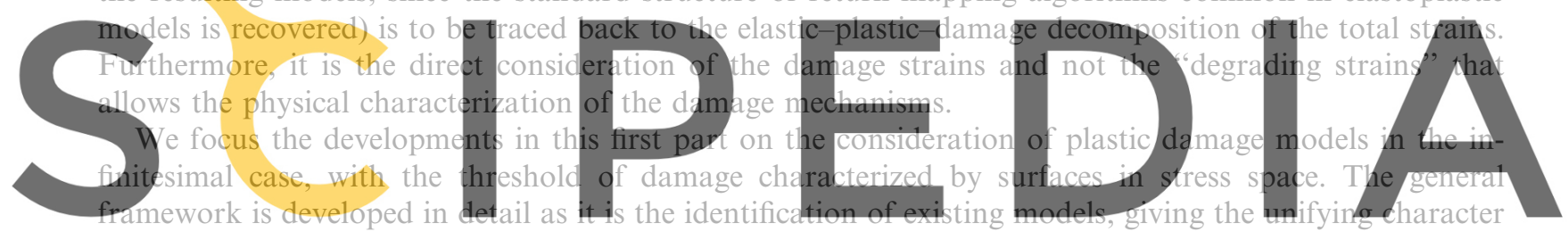

described above. We present in Part II, the application of these ideas to the formulation of a simple plastic

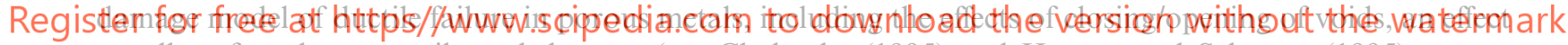
usually referred to as unilateral damage (see Chaboche (1995) and Hansen and Schreyer (1995) among others). Numerical algorithms for the integration of the plastic damage framework investigated herein can also be found in Part II.

An outline of the rest of the paper is as follows. Section 2 develops the general framework proposed in this work. Emphasis is given to the incorporation of multiple damage mechanisms in the inelastic response of the material, hence illustrating the ability of the proposed formulation to handle the physical characterization of each of them independently. A completely consistent thermodynamic framework is considered for a particular damage mechanism. Section 3 particularizes the preceding developments to a quadratic damage potential. We show in the different subsections of Section 3.1 that this case includes as particular cases existing damage models from isotropic damage to smeared crack models. Finally, Section 4 concludes with some final remarks.

\section{General formulation}

We develop in this section, the general framework for the formulation of damage theories proposed in this work. The fundamental kinematic assumptions underlying the proposed framework are presented in Section 2.1 with the resulting evolution equations of the considered internal variables described in Section 2.3 after deriving the dissipation expression in Section 2.2. The tangent rate equations are derived in Section 2.4 . 


\subsection{Fundamental kinematic assumptions}

With the assumption of infinitesimal deformations considered herein, the kinematics of the deformation at a given point $\boldsymbol{x}$ of a solid $\Omega$ (identified with its reference placement through the above assumption) is characterized, under the classical assumption of local response, by the infinitesimal strain tensor

$$
\boldsymbol{\varepsilon}:=\operatorname{sym}[\nabla \boldsymbol{u}] \in \mathscr{S},
$$

for the displacement field $\boldsymbol{u}: \Omega \rightarrow \mathbb{R}^{n_{\mathrm{dim}}}\left(n_{\mathrm{dim}}=1,2\right.$ or 3 ) and spatial gradient $\nabla(\cdot)$. We denote by $\mathscr{S}$, the linear space of symmetric $n_{\mathrm{dim}} \times n_{\mathrm{dim}}$ tensors. In this context, we introduce the fundamental decomposition of the total strain tensor $\varepsilon$ in

$$
\boldsymbol{\varepsilon}=\boldsymbol{\varepsilon}^{\mathrm{e}}+\boldsymbol{\varepsilon}^{\mathrm{p}}+\boldsymbol{\varepsilon}^{\mathrm{d}}
$$

that is, in elastic $\varepsilon^{\mathrm{e}}$, plastic $\varepsilon^{\mathrm{p}}$ and damage $\varepsilon^{\mathrm{d}}$ parts. The latter is assumed decomposed additively as

that is, as the sum of $n_{\text {dam }}$ similar terms.

Additive kinematic decompositions of the forms (2.2) and (2.3) are commonly used in the context of elastoplastic theories, but are rarely considered in the context of damage models. It is the main aim of this paper to explore this decomposition in this latter case, and to show the convenience of their use from the

point of view of the modelin

gration. In particular, the

Fig. 1 illustrates the basic idea behind the assumed decomposition (2.3) through a typical uniaxial

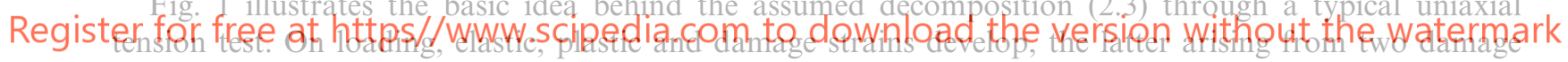

mechanisms in the material, say, crack and void formation. In more general situations, the different damage mechanisms may also be identified with different crack orientations at a given material point, brittie damage associated to particularly oriented fibers embedded in the material, etc., i.e., with different physical mechanisms introducing a damage response in the material under repeated loading and unloading. In

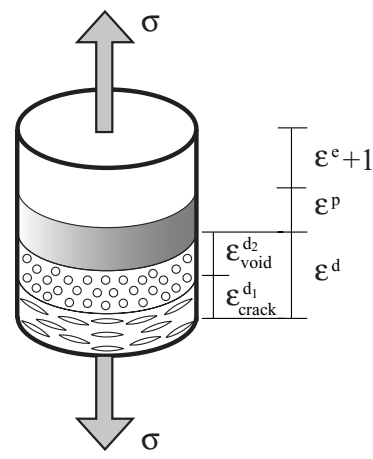

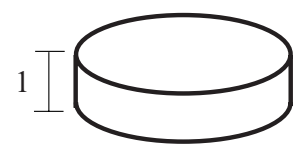
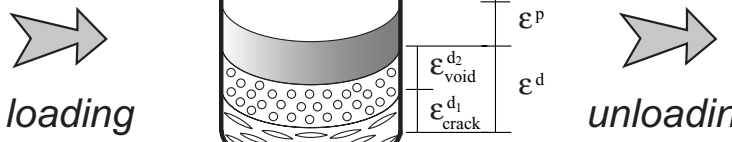

unloading

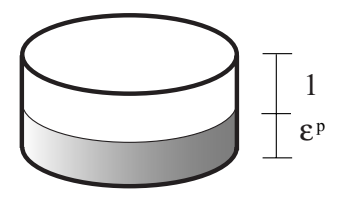

Fig. 1. Sketch of a uniaxial tension test of a material exhibiting elastic, plastic and damage strains, the latter consisting of the addition of two damage mechanisms, say the formation of cracks and voids. On unloading, both the elastic and damage strains are recovered. 
contrast with the plastic part $\varepsilon^{\mathrm{p}}$, the damage strain $\varepsilon^{\mathrm{d}}$ is thought as a recoverable strain, in the sense that it is recovered upon unloading, a feature that will become more apparent when discussing the stored energy of the material in Section 2.2. The decomposition (2.3) allows the modeling of each component of the material's response, i.e., elastic, plastic and damage components based on the corresponding strains, with the explicit consideration of the possible several couplings between these components, if necessary. Fig. 2 illustrates a typical stress/strain relation in this uniaxial setting, as discussed in detail in Section 2.2 when formulating a general thermodynamic framework of the material response.

As illustrated in Section 3, the damage mechanisms characteristic of the response of many materials lead to particular constrained forms of the associated damage strains $\boldsymbol{\varepsilon}^{\mathrm{d}}$; typical examples involve volumetric damage strains, rank-one strains associated to cracks in brittle materials, etc. In general, and for each damage mechanism $\left(d_{\mathrm{I}}=1, n_{\mathrm{dam}}\right)$, we have $\boldsymbol{\varepsilon}^{d_{\mathrm{I}}} \in \mathscr{V}^{d_{\mathrm{I}}}$ for the linear subspace

$$
\mathscr{N}^{d_{1}}=\left\{\varepsilon^{d_{1}}=\sum_{a=1}^{n_{d_{\mathrm{I}}}} \mathbb{P}_{a}^{d_{1}} e_{a}^{d_{\mathrm{I}}} \quad \text { for } e_{a}^{d_{\mathrm{I}}} \in \mathbb{R} \text { and } \mathbb{P}_{a}^{d_{1}} \in \mathscr{S}\right\} \subset \mathscr{S},
$$

where the set of symmetric tensors $\left\{\mathbb{P}_{a}^{d_{1}}\right\}_{a=1}^{n_{d_{1}}}$ is assumed to define a basis of $\mathscr{f}^{d_{\mathrm{I}}}$, with dimension $n_{d_{1}} \leqslant\left(n_{\mathrm{dim}}+1\right) n_{\mathrm{dim}} / 2$. Without loss of generality, we consider an orthonormal basis in the usual inner product of symmetric tensors $\mathscr{S}$ (i.e., double contraction $A: A:=A_{i j} A_{i j}$, summation implied). In this way, we have the orthogonality relations
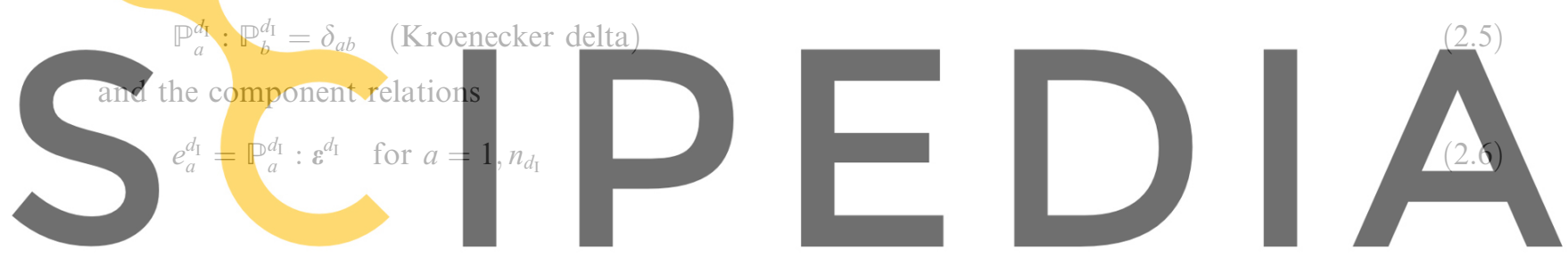

Register for free at https//www.scipedia.com to download the version without the watermark

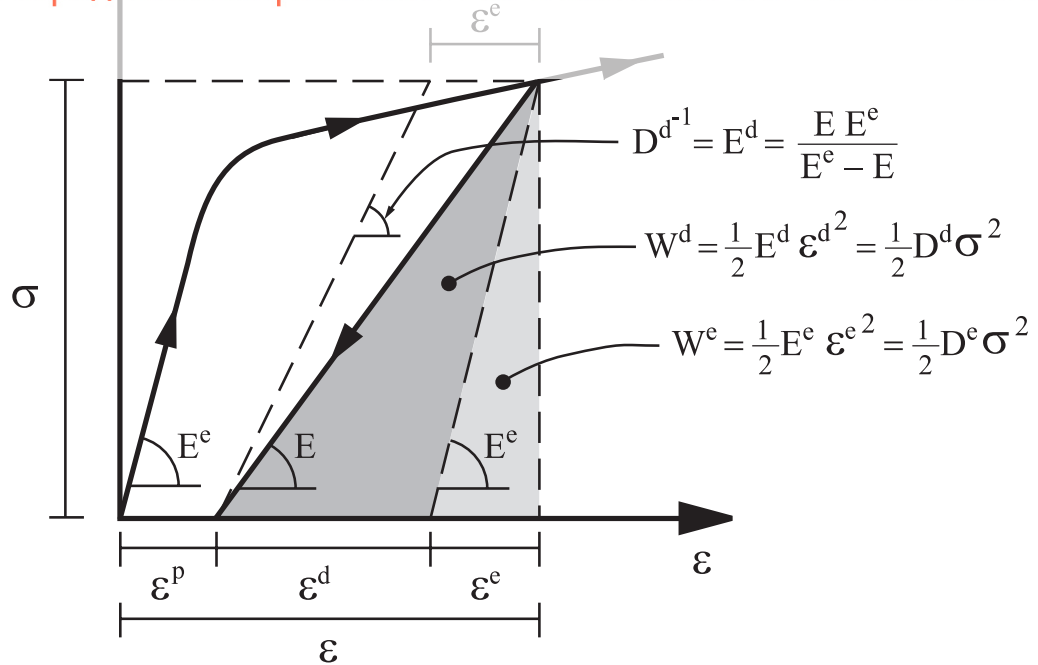

Fig. 2. Typical stress-strain relation of a plastic damage material with quadratic elastic and damage potentials under uniaxial loading conditions. The three different components of the strain, i.e., elastic $\varepsilon^{\mathrm{e}}$, plastic $\boldsymbol{\varepsilon}^{\mathrm{p}}$, and damage strains $\boldsymbol{\varepsilon}^{\mathrm{d}}$ are identified. We also observe the representation of the different axial moduli for the current $E$, (initial) elastic $E^{\mathrm{e}}$ and damaged $E^{\mathrm{d}}=E E^{\mathrm{e}} /\left(E-E^{\mathrm{e}}\right)$ Young moduli, with the corresponding compliance $D^{\mathrm{d}}=E^{\mathrm{d}^{-1}}$, employed in the assumed linear loading/unloading response. 
for each damage mechanism $d_{\mathrm{I}}=1, n_{\mathrm{dam}}$ independently. To avoid the use of double indices when referring to the damage mechanism and damage strain component, we write the component relation in Eq. (2.4) in the compact form

$$
\boldsymbol{\varepsilon}^{d_{\mathrm{I}}}=\mathbb{P}^{d_{\mathrm{I}}^{\mathrm{T}}} \boldsymbol{e}^{d_{\mathrm{I}}} \quad \text { for } \boldsymbol{e}^{d_{\mathrm{I}}}:=\left[e_{a}^{d_{\mathrm{I}}}\right]=\left[\begin{array}{c}
e_{1}^{d_{\mathrm{I}}} \\
\vdots \\
e_{n_{d_{\mathrm{I}}}}^{d_{\mathrm{I}}}
\end{array}\right]
$$

and $\mathbb{P}^{d_{\mathrm{I}}}=\left[\mathbb{P}_{a(i j)}^{d_{\mathrm{I}}}\right]$ for $a=1, n_{d_{\mathrm{I}}}$ and $i, j=1, n_{\mathrm{dim}}$, with $\mathbb{P}_{\mathrm{I}}^{d_{\mathrm{I}}^{\mathrm{T}}}:=\left[\mathbb{P}_{(i j) a}^{d_{\mathrm{I}}}\right]$. We write symbolically

$$
\mathbb{P}^{d_{\mathrm{I}}}=\left[\begin{array}{c}
\mathbb{P}^{d_{\mathrm{I}}} \\
1 \\
\vdots \\
\mathbb{P}_{d_{\mathrm{I}}} \\
n_{d_{\mathrm{I}}}
\end{array}\right]
$$

\section{in the developments that follow.}

\section{Remark 2.1.}

1. In the numerical implementation, we use the standard vector notation $\varepsilon=\left\{\begin{array}{lll}\varepsilon_{11} & \varepsilon_{22} & 2 \varepsilon_{12}\end{array}\right\}^{\mathrm{T}}$ and $\sigma=\left\{\begin{array}{lll}\sigma_{11} & \sigma_{22} & \sigma_{12}\end{array}\right\}^{\mathrm{T}}$ for, e.g., plane problems. The linear operator $\mathbb{P}^{n_{d_{1}}}$ corresponds to a $4 n_{d_{1}}$ vector $\left(6 n_{d_{\mathrm{I}}}\right.$ in three-dimensional problems in this notation).

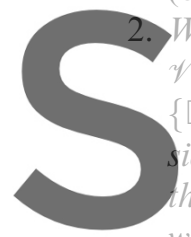

We have assumed, for simplicity, that the projection ma

d as a nonlinear manifo

$\left.\mathbb{P}_{a}^{d_{1}}\right\}_{a=1}^{n_{d_{1}}}$ at a given poin

ider only the simple case $f$

he damage space is treated separate.

rork (Armero and Oller 1999) for the inposition of

voids in a particular coupled plastic damage model of porous metals.

Register for free at https//www.scipedia.com to download the version without the watermark

\subsection{Elastic energy and plastic-damage dissipation}

The considerations described in Section 2.1 identified the kinematics of the problem of interest. In particular, we considered the existence of $n_{\text {dam }}$ mechanisms leading to the damage of the solid. In accordance, we introduce the decomposition of the stored energy function,

$$
W=W^{\mathrm{e}}\left(\boldsymbol{\varepsilon}^{\mathrm{e}}\right)+\mathscr{H}^{\mathrm{p}}\left(\mathscr{I}^{\mathrm{p}}\right)+\sum_{d_{\mathrm{I}}=1}^{n_{\mathrm{dam}}} W^{d_{\mathrm{I}}}\left(\boldsymbol{\varepsilon}^{d_{\mathrm{I}}} ; \mathscr{I}^{d_{\mathrm{I}}}\right),
$$

for a stored energy function $W^{\mathrm{e}}(\cdot)$ characterizing the elastic response of the solid in terms of the elastic strains $\boldsymbol{\varepsilon}^{\mathrm{e}}$, a potential $\mathscr{H}^{\mathrm{p}}(\cdot)$ modeling the hardening plastic response in terms of a general set of plastic internal variables $\mathscr{I}^{\mathrm{p}}$ (not including $\varepsilon^{\mathrm{p}}$ ), and a set of stored energy functions $W^{d_{\mathrm{I}}}(\cdot)$ characterizing the internal energy stored in each of the $n_{\mathrm{dam}}$ damage mechanisms. The latter is given in terms of the corresponding damage strain $\varepsilon^{d_{1}}$ and a general set of internal variables $\mathscr{I}^{d_{1}}$ (not including $\varepsilon^{d_{1}}$ ) characterizing the inelastic response associated to the damage mechanisms. For simplicity, we assume a full decoupling of the different damage mechanisms, leading to an independent set of internal variables for each of them. Uncoupled thermomechanical conditions have been assumed with the identification of the stored energy function $W$ with the Helmholtz free energy of the material in the case of isothermal deformations as it is customary. 
A main feature of the considered formulation is the presence of the damage potential $W^{d_{I}}(\cdot)$ in terms of the damage strains $\boldsymbol{\varepsilon}^{d_{\mathrm{I}}}$ and the general set of internal variables $\mathscr{I}^{d_{\mathrm{I}}}$. A simple choice for this potential is provided by a quadratic potential, as considered in detail in Section 3, which together with a quadratic elastic potential $W^{\mathrm{e}}(\cdot)$ leads to a linear response of the material during unloading and subsequent reloading. The stiffness or, equivalently, the compliance associated to the damage of the material as observed in these processes appears as a natural choice for one of the internal damage variables $\mathscr{I}^{d_{1}}$. Fig. 2 illustrates these ideas for the simple uniaxial tension test depicted in Fig. 1 for one damage mechanism. Alternative forms of this damage potential can be assumed in general. We observe again in Fig. 2, the recoverable nature of the damage strains $\boldsymbol{\varepsilon}^{\mathrm{d}}$ and the corresponding damage potential upon unloading, a direct consequence of the explicit consideration of these strains in the expression of the stored energy function, as it occurs with the elastic strain $\boldsymbol{\varepsilon}^{\mathrm{e}}$ and in contrast with the plastic strains $\boldsymbol{\varepsilon}^{\mathrm{p}}$.

Given the expression (2.9) of the stored energy of the material, the local dissipation rate (Simo and Hughes, 1998) can be written, after using the kinematic decomposition (2.2), as

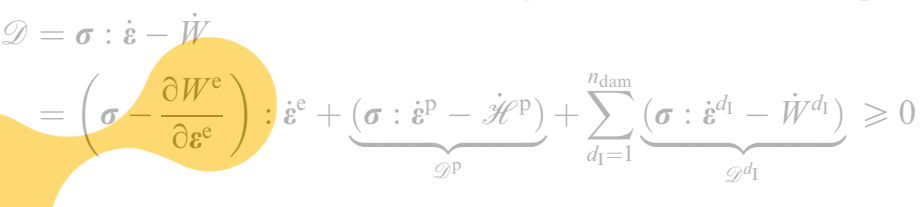

for the stress tensor $\sigma$. After imposing the physically motivated constraints

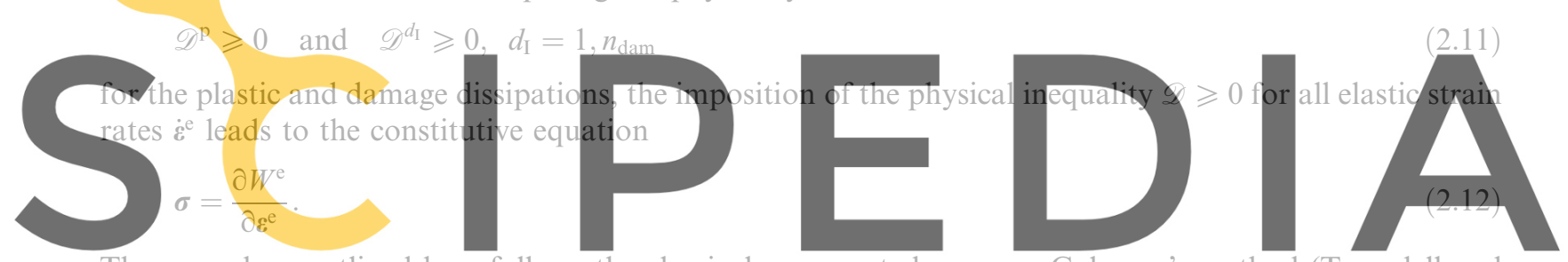

The procedure outlined here follows the classical arguments known as Coleman's method (Truesdell and Registerfor free at https 199 ). www.scipedia.com to download the version without the watermark

$$
\mathscr{D}^{\mathrm{p}}=\boldsymbol{\sigma}: \dot{\boldsymbol{\varepsilon}}^{\mathrm{p}}+\mathscr{Q}^{\mathrm{p}} \odot \mathscr{\mathscr { I }}^{\mathrm{p}} \geqslant 0 \text { for } \mathscr{Q}^{\mathrm{p}}:=-\frac{\partial \mathscr{H}^{\mathrm{p}}}{\partial \mathscr{I}^{\mathrm{p}}},
$$

the conjugate plastic hardening variable. The symbol $\odot$ in expression (2.13) denotes a general tensor contraction depending on the character of the internal variables $\mathscr{I}^{\mathrm{p}}$. The specific case of a scalar variable $\mathscr{I}^{\mathrm{p}} \equiv \alpha^{\mathrm{p}}$ with scalar conjugate variable $\mathscr{2}^{\mathrm{p}} \equiv q^{\mathrm{p}}$ and standard scalar multiplication between them, is considered in the particular model example of Section 3.

The damage dissipation $\mathscr{D}^{d_{\mathrm{I}}}$ in Eq. (2.10) can be expressed in the same way as

$$
\mathscr{D}^{d_{\mathrm{I}}}=\left(\boldsymbol{\sigma}-\frac{\partial W^{d_{\mathrm{I}}}}{\partial \boldsymbol{\varepsilon}^{d_{\mathrm{I}}}}\right): \dot{\boldsymbol{\varepsilon}}^{d_{\mathrm{I}}}-\frac{\partial W^{d_{\mathrm{I}}}}{\partial \mathscr{I}^{d_{\mathrm{I}}}} \odot \dot{\mathscr{I}}^{d_{\mathrm{I}}} \geqslant 0
$$

where $\odot$ denotes again a general contraction depending on the character of the internal variables $\mathscr{I}^{d_{1}}$. Following the same arguments as before, i.e., imposing the non-negative character of the dissipation (2.14) for independent variations of the damage strains $\boldsymbol{\varepsilon}^{d_{1}}$ and internal variables $\mathscr{I}^{d_{1}}$, leads after noting the constrained character of Eq. (2.14) (since $\varepsilon^{d_{\mathrm{I}}} \in \mathscr{V}^{d_{\mathrm{I}}}$ ) to

$$
\begin{aligned}
& \mathscr{D}^{d_{\mathrm{I}}}=\mathscr{2}^{d_{\mathrm{I}}} \odot \dot{\mathscr{I}}^{d_{\mathrm{I}}} \geqslant 0 \\
& \text { for } \mathscr{2}^{d_{\mathrm{I}}}:=-\frac{\partial W^{d_{\mathrm{I}}}}{\partial \mathscr{I}^{d_{\mathrm{I}}}}
\end{aligned}
$$


and the general relation

$$
\boldsymbol{\sigma}=\frac{\partial W^{d_{\mathrm{I}}}}{\partial \boldsymbol{\varepsilon}^{d_{\mathrm{I}}}}+\boldsymbol{\sigma}^{\perp}
$$

for a component $\boldsymbol{\sigma}^{\perp} \in \mathscr{V}^{d_{\mathrm{I}}^{\perp}}$ defined by

$$
\mathscr{V}^{d_{\mathrm{I}}^{\perp}}:=\left\{\boldsymbol{\sigma} \in \mathscr{S}: \quad \boldsymbol{\sigma}: \boldsymbol{\varepsilon}^{d_{\mathrm{I}}}=0 \quad \forall \boldsymbol{\varepsilon}^{d_{\mathrm{I}}} \in \mathscr{V}^{d_{\mathrm{I}}}\right\}
$$

that is, the orthogonal complement of the original space of damage strains $\mathscr{V}^{d_{\mathrm{I}}}$. The explicit absence of the damage strains $\boldsymbol{\varepsilon}^{d_{\mathrm{I}}}$ in the expression of the damage dissipation (2.15a) emphasizes once more the recoverable character of these strains, in contrast with $\varepsilon^{\mathrm{p}}$.

If $\sigma^{d_{1}}$ denotes the projection of the general stress tensor $\sigma \in \mathscr{S}$ onto the space $\mathscr{V}^{d_{1}}$, we can write the general relation

$$
\begin{gathered}
\sigma^{d_{1}}=\sum_{a=1}^{n_{d_{1}}} \mathbb{P}_{a}^{d_{1}} s_{a}^{d_{1}} \\
\text { for } s_{a}^{d_{1}}:=\mathbb{P}_{a}^{d_{1}}: \sigma
\end{gathered}
$$

or, simply,
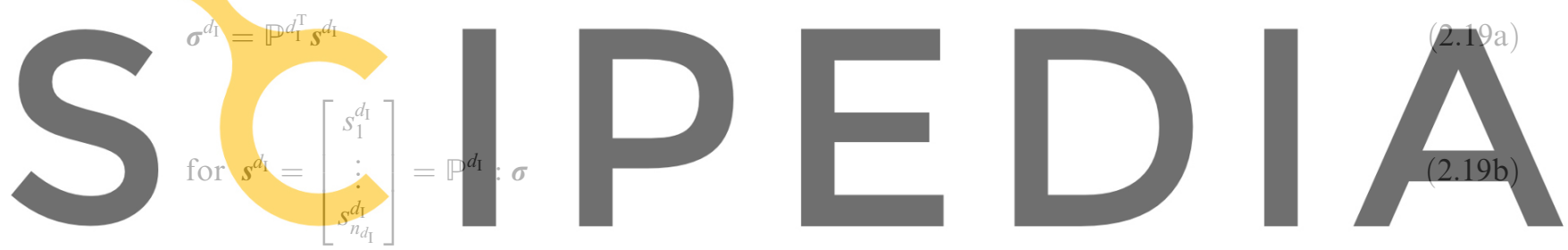

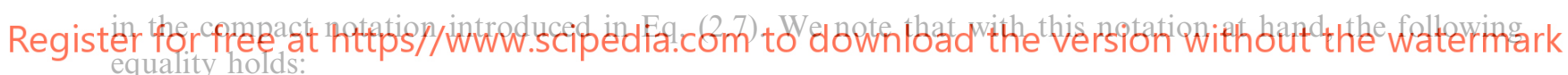

$$
\boldsymbol{\sigma}^{d_{1}}: \boldsymbol{\varepsilon}^{d_{1}}=\boldsymbol{s}^{d_{1}} \cdot \boldsymbol{e}^{d_{1}} \quad \forall \boldsymbol{\sigma}^{d_{1}}=\mathbb{P}^{d_{\mathrm{i}}^{\mathrm{T}}} \boldsymbol{s}^{d_{1}}, \quad \boldsymbol{\varepsilon}^{d_{1}}=\mathbb{P}^{d_{\mathrm{T}}^{\mathrm{T}}} \boldsymbol{e}^{d_{1}} \in \mathscr{V}^{d_{1}}
$$

given the orthogonality relation (2.5). Projecting the relation (2.16) on $\mathscr{V}^{d_{1}}$, we have

$$
\boldsymbol{\sigma}^{d_{\mathrm{I}}}=\frac{\partial W^{d_{\mathrm{I}}}}{\partial \boldsymbol{\varepsilon}^{d_{\mathrm{I}}}}
$$

or, simply,

$$
\boldsymbol{s}^{d_{\mathrm{I}}}=\frac{\partial \widehat{W}^{d_{\mathrm{I}}}}{\partial \boldsymbol{e}^{d_{\mathrm{I}}}} \quad \text { or, in components, } \quad s_{a}^{d_{\mathrm{I}}}=\frac{\partial \widehat{W}^{d_{\mathrm{I}}}}{\partial e_{a}^{d_{\mathrm{I}}}} \quad\left(a=1, n_{d_{\mathrm{I}}}\right),
$$

where

$$
\widehat{W}^{d_{\mathrm{I}}}\left(\boldsymbol{e}^{d_{\mathrm{I}}} ; \mathscr{I}^{d_{\mathrm{I}}}\right)=W^{d_{\mathrm{I}}}\left(\boldsymbol{\varepsilon}^{d_{\mathrm{I}}} ; \mathscr{I}^{d_{\mathrm{I}}}\right) \quad \text { for } \boldsymbol{\varepsilon}^{d_{\mathrm{I}}}=\mathbb{P}^{d_{\mathrm{I}}^{\mathrm{T}}} \boldsymbol{e}^{d_{\mathrm{I}}} \in \mathscr{V}^{d_{\mathrm{I}}}
$$

after using the compact notation introduced in Eq. (2.7). We also note the relation

$$
\mathscr{2}^{d_{\mathrm{I}}}=-\frac{\partial W^{d_{\mathrm{I}}}}{\partial \mathscr{I}^{d_{\mathrm{I}}}}=-\frac{\partial \widehat{W}^{d_{\mathrm{I}}}}{\partial \mathscr{I}^{d_{\mathrm{I}}}}
$$

for future use. 
Remark 2.2. We emphasize that the formal argument behind the separate treatment in Eq. (2.11) of the different components of the dissipation does not imply that these components will only occur independently. The total dissipation is given by Eq. (2.10), allowing deformation paths that involve coupled plastic and damage dissipation. We observe in this respect that the presence of a common stress in the two components of the dissipation does indeed lead to this coupling. The "equilibrium" relation (2.18b), identifying the stress components in the different dissipative mechanisms, appears then as fundamental. In fact, this role is emphasized in the numerical treatment developed in Part II of this work, since it becomes the key relation for an efficient integration of the resulting plastic damage models. We also refer to Section 3.2.1, where the dissipation (2.10) is recast in the context of a specific model involving the classical notion of effective stresses.

\subsection{The plastic damage evolution equations}

The above thermodynamic framework leads directly to the evolution equations of the damage internal variables $\mathscr{I}^{d_{1}}$ and plastic variables $\left\{\varepsilon^{\mathrm{p}}, \mathscr{I}^{\mathrm{p}}\right\}$. In particular, we note from the expressions (2.13) and (2.15a), the conjugate character of the internal variables $\mathscr{2}^{d_{1}}$ and $\mathscr{I}^{d_{1}}$ for the damage part and $\left\{\varepsilon^{\mathrm{p}}, \mathscr{I}^{\mathrm{p}}\right\}$ with $\left\{\boldsymbol{\sigma}, \mathscr{2}^{\mathrm{p}}\right\}$ for the plastic part. In this context, we introduce a set of damage surfaces $\phi^{d_{1}}\left(2^{d_{I}}\right)$ for each damage mechanism $d_{1}=1, n_{\mathrm{dam}}$ and a plastic yield surface $\phi^{\mathrm{p}}\left(\sigma, \mathscr{I}^{\mathrm{p}}\right)$ by the relations

$\phi^{d_{\mathrm{I}}}\left(2^{d_{\mathrm{I}}}\right) \leqslant 0$,
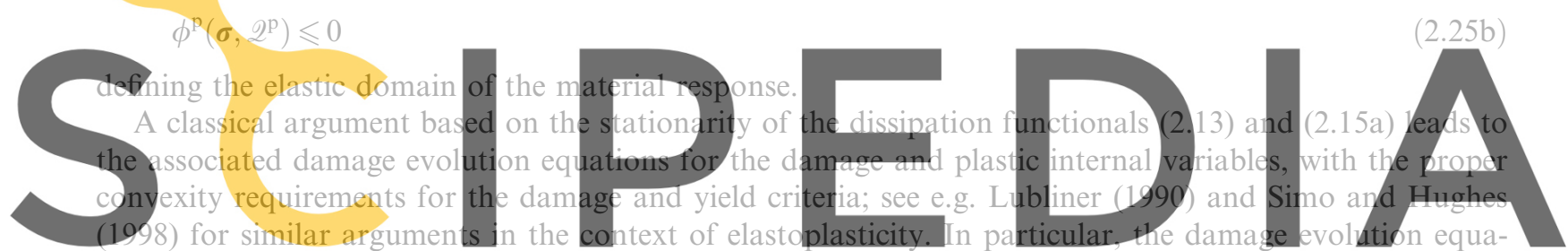

tions read

Register for free at $_{\phi}$ https//www.scipedia.com to download the version without the watermark

for the damage multiplier $\gamma^{d_{I}}$ satisfying the complementary Kuhn-Tucker loading/unloading conditions

$$
\gamma^{d_{\mathrm{I}}} \geqslant 0, \quad \phi^{d_{\mathrm{I}}} \leqslant 0 \quad \text { and } \quad \gamma^{d_{\mathrm{I}}} \phi^{d_{\mathrm{I}}}=0
$$

as well as the damage consistency condition

$$
\gamma^{d_{1}} \dot{\phi}^{d_{1}}=0
$$

during persistent damage. Similarly, the associated plastic evolution equations read

$$
\begin{aligned}
& \dot{\boldsymbol{\varepsilon}}^{\mathrm{p}}=\gamma^{\mathrm{p}} \frac{\partial \phi^{\mathrm{p}}}{\partial \boldsymbol{\sigma}}, \\
& \dot{\mathscr{I}}^{\mathrm{p}}=\gamma^{\mathrm{p}} \frac{\partial \phi^{\mathrm{p}}}{\partial \mathscr{Q}^{\mathrm{p}}}
\end{aligned}
$$

for the plastic multiplier $\gamma^{\mathrm{p}}$ satisfying

$$
\gamma^{\mathrm{p}} \geqslant 0, \quad \phi^{\mathrm{p}} \leqslant 0 \quad \text { and } \quad \gamma^{\mathrm{p}} \phi^{\mathrm{p}}=0
$$

and the plastic consistency condition

$$
\gamma^{\mathrm{p}} \dot{\phi}^{\mathrm{p}}=0 .
$$


Table 1

Summary of the constitutive relations for a general damage model

(1) Elastic stress-strain relation

$\boldsymbol{\sigma}=\frac{\partial W^{\mathrm{e}}}{\partial \boldsymbol{\varepsilon}^{\mathrm{e}}}$

for an elastic potential $W^{\mathrm{e}}\left(\varepsilon^{\mathrm{e}}\right)$.

(2) Damage stress-strain relations

$\boldsymbol{\sigma}^{d_{\mathrm{I}}}=\frac{\partial W^{d_{\mathrm{I}}}}{\partial \boldsymbol{\varepsilon}^{d_{\mathrm{I}}}} \quad$ or $\quad \boldsymbol{\varepsilon}^{d_{\mathrm{I}}}=\frac{\partial \chi^{d_{\mathrm{I}}}}{\partial \boldsymbol{\sigma}^{d_{\mathrm{I}}}} \quad\left(\Longleftrightarrow \boldsymbol{s}^{d_{\mathrm{I}}}=\frac{\partial \widehat{W}^{d_{\mathrm{I}}}}{\partial \boldsymbol{e}^{d_{\mathrm{I}}}} \quad\right.$ or $\left.\quad \boldsymbol{e}^{d_{\mathrm{I}}}=\frac{\partial \widehat{\chi}^{d_{\mathrm{I}}}}{\partial \boldsymbol{s}^{d_{\mathrm{I}}}}\right)$

for the conjugate damage potentials $W^{d_{1}}\left(\boldsymbol{\varepsilon}^{d_{1}} ; \mathscr{I}^{d_{1}}\right)$ and $\chi^{d_{\mathrm{I}}}\left(\boldsymbol{\sigma}^{d_{1}} ; \mathscr{I}^{d_{1}}\right)$ (or, equivalently, $\widehat{W}^{d_{\mathrm{I}}}\left(\boldsymbol{e}^{d_{\mathrm{I}}} ; \mathscr{I}^{d_{\mathrm{I}}}\right)$ and $\widehat{\chi}^{d_{\mathrm{I}}}\left(\boldsymbol{s}^{d_{\mathrm{I}}} ; \mathscr{I}^{d_{\mathrm{I}}}\right)$ ), for each damage mechanism $d_{\mathrm{I}}=1, n_{\mathrm{dam}}$. The damage stresses $\boldsymbol{\sigma}^{d_{\mathrm{I}}}$ are given in terms of the (total) stresses $\boldsymbol{\sigma}$ by the projection relation (equilibrium)

$\sigma^{d_{1}}=\sum_{a=1}^{n_{d_{\mathrm{I}}}} \mathbb{P}_{a}^{d_{1}} s_{a}^{d_{1}} \quad$ with $s_{a}^{d_{1}}=\mathbb{P}_{a}^{d_{1}}: \sigma$

(3) The (associated) damage evolution equations
(3) stresses.

$\left\{\begin{array}{l}\dot{\mathscr{I}}^{d_{1}}=\gamma^{d_{1}} \frac{\partial \phi^{d_{1}}}{\partial 2^{d_{1}}}, \\ \gamma^{d_{1}} \geqslant 0, \quad \phi^{d_{1}} \leqslant 0, \quad \gamma^{d_{1}} \phi^{d_{1}}=0 \\ d_{1} \dot{\phi}^{d_{1}}=0\end{array}\right.$

where $2^{d_{1}}=-\partial \widehat{W}^{d_{1}} / \partial \mathscr{I}^{d_{1}}=\partial \widehat{\chi}^{d_{1}} / \partial \mathscr{I}^{d_{1}}$, for each damage mechanism $d_{1}=1, n_{\text {dam }}$
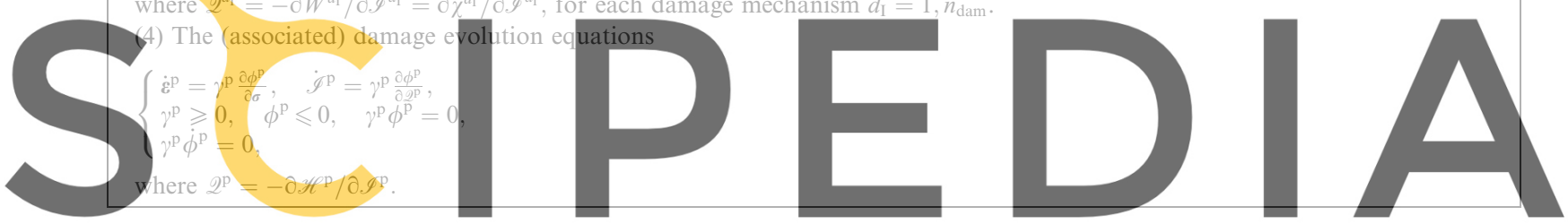

The above relations define completely the plastic damage model A summary of these constitntive relations
Register or tre

Remark 2.3. Additional extensions can be easily accommodated in the above framework. For example, the consideration of general damage and plastic potentials in the damage and plastic evolution equations (2.26) and (2.29), different from the damage and plastic surfaces, respectively, may be used in the formulation of nonassociated models. Similarly, the formulation of viscous damage models is easily accomplished by the viscous regularization of the Perzyna type as employed in elastoplasticity, namely, replacing the Kuhn-Tucker loading and unloading conditions (2.27) and consistency condition (2.28) by the evolution equation

$$
\gamma^{d_{1}}=\frac{\left\langle f^{d_{1}}\left(\phi^{d_{1}}\right)\right\rangle}{\eta^{d_{\mathrm{I}}}}
$$

for a damage viscosity $\eta^{d_{1}}$, general monotonically increasing scalar function $f^{d_{\mathrm{I}}}(\cdot)$, and Macaulay brackets $\langle x\rangle:=(x+|x|) / 2$ while retaining the evolution equation (2.26). Finally, the case of multi-surface damage mechanisms can be easily considered through a damage evolution equation of the Koiter type (Simo and Hughes, 1998), following the same arguments as in elastoplasticity. Details are omitted.

\subsubsection{Damage models in stress space}

The above arguments led to the consideration of damage surfaces defined in terms of the internal variables $2^{d_{1}}$. These variables are defined by Eq. $(2.15 \mathrm{~b})$ and, thus, they can be written as

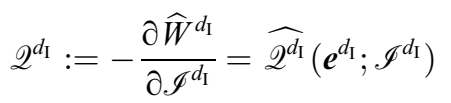


that is, as functions of the damage strains $\boldsymbol{e}^{d_{\mathrm{I}}}$ (or, equivalently, $\boldsymbol{\varepsilon}^{d_{1}}$ ) and conjugate internal variables $\mathscr{I}^{d_{\mathrm{I}}}$. However, and as noted in Section 1, our interest in this paper is the consideration of stress-based damage models, that is, with the damage surfaces $\phi^{d_{I}}$ in terms of the stresses $\sigma$. For this purpose, the internal variables $2^{d_{1}}$ can be alternatively defined as follows.

We introduce the damage complementary energy function, denoted for the mechanism $d_{\mathrm{I}}=1$, $n_{\mathrm{dam}}$ by

$$
\chi^{d_{\mathrm{I}}}\left(\boldsymbol{\sigma}^{d_{\mathrm{I}}} ; \mathscr{I}^{d_{\mathrm{I}}}\right)=\widehat{\chi}^{d_{\mathrm{I}}}\left(\boldsymbol{s}^{d_{1}} ; \mathscr{I}^{d_{\mathrm{I}}}\right) \quad \text { for } \boldsymbol{\sigma}^{d_{\mathrm{I}}}=\mathbb{P}^{d_{\mathrm{I}}^{\mathrm{T}}} \boldsymbol{s}^{d_{1}} \in \mathscr{V}^{d_{\mathrm{I}}}
$$

and obtained by the Legendre transform of the stored energy function $W^{d_{1}}$ in the damage strains. That is, for a given stress $\boldsymbol{\sigma}^{d_{1}}=\mathbb{P}^{d_{1}^{\mathrm{T}}} \boldsymbol{s}^{d_{\mathrm{I}}} \in \mathscr{V}^{d_{\mathrm{I}}}$, we define the function

$$
\begin{aligned}
\widehat{\chi}^{\mathrm{d}}\left(\boldsymbol{s}^{d_{\mathrm{I}}} ; \mathscr{I}^{d_{\mathrm{I}}}\right): & =\max _{\boldsymbol{e}^{d_{\mathrm{I}}}}\{\underbrace{\boldsymbol{s}^{d_{\mathrm{I}}} \cdot \boldsymbol{e}^{d_{\mathrm{I}}}}_{\sigma^{d_{\mathrm{I}}}: g^{d_{\mathrm{I}}}}-\widehat{W}^{d_{\mathrm{I}}}\left(\boldsymbol{e}^{d_{\mathrm{I}}} ; \mathscr{I}^{d_{\mathrm{I}}}\right)\} \\
& =\boldsymbol{s}^{d_{\mathrm{I}}}: \widehat{\boldsymbol{e}^{d_{\mathrm{I}}}}\left(\boldsymbol{s}^{d_{\mathrm{I}}}, \mathscr{I}^{d_{\mathrm{I}}}\right)-\widehat{W}^{d_{\mathrm{I}}}\left(\widehat{\boldsymbol{e}^{d_{\mathrm{I}}}}\left(\boldsymbol{s}^{d_{\mathrm{I}}}, \mathscr{I}^{d_{\mathrm{I}}}\right) ; \mathscr{I}^{d_{\mathrm{I}}}\right)
\end{aligned}
$$

for the function $\widehat{\boldsymbol{e}^{d_{1}}}\left(\boldsymbol{s}^{d_{1}}, \mathscr{I}^{d_{1}}\right)$ obtained by inverting the constitutive relation

$$
\boldsymbol{s}^{d_{1}}=\frac{\partial \widehat{W}^{d_{\mathrm{I}}}}{\partial \boldsymbol{e}^{d_{\mathrm{I}}}}\left(\boldsymbol{e}^{d_{\mathrm{I}}} ; \mathscr{I}^{d_{\mathrm{I}}}\right) .
$$

A simple argument based on the chain rule applied to Eq. (2.36) shows that

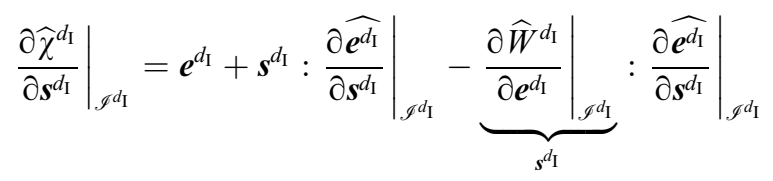

so

$$
\boldsymbol{e}^{d_{\mathrm{I}}}=\frac{\partial \widehat{\chi}^{d_{\mathrm{I}}}}{\partial \boldsymbol{s}^{d_{\mathrm{I}}}} \quad \text { or, in components, } \quad e_{a}^{d_{\mathrm{I}}}=\frac{\partial \widehat{\chi}^{d_{\mathrm{I}}}}{\partial s_{a}^{d_{\mathrm{I}}}}\left(a=1, n_{d_{\mathrm{I}}}\right),
$$

where the arguments of the function $\widehat{\chi}^{d_{\mathrm{I}}}\left(\boldsymbol{s}^{d_{\mathrm{I}}} ; \mathscr{I}^{d_{\mathrm{I}}}\right)$ are implied. Equivalently, we write

$$
\boldsymbol{\varepsilon}^{d_{\mathrm{I}}}=\frac{\partial \chi^{d_{\mathrm{I}}}}{\partial \boldsymbol{\sigma}^{d_{\mathrm{I}}}}
$$

for $\chi^{d_{\mathrm{I}}}\left(\boldsymbol{\sigma}^{d_{1}} ; \mathscr{I}^{d_{1}}\right)$ defined in Eq. (2.34).

A calculation similar to Eq. (2.38) leads easily to the relation

$$
\left.\frac{\partial \widehat{\chi}^{d_{\mathrm{I}}}}{\partial \mathscr{I}^{d_{\mathrm{I}}}}\right|_{s^{d_{\mathrm{I}}}}=-\left.\frac{\partial \widehat{W}^{d_{\mathrm{I}}}}{\partial \mathscr{I}^{d_{\mathrm{I}}}}\right|_{e^{d_{\mathrm{I}}}},
$$

thus defining the internal variables

$$
\mathscr{2}^{d_{\mathrm{I}}}=\widehat{\mathscr{2}^{d_{\mathrm{I}}}}\left(\boldsymbol{s}^{d_{\mathrm{I}}} ; \mathscr{I}^{d_{\mathrm{I}}}\right)=\frac{\partial \widehat{\chi}^{d_{\mathrm{I}}}}{\partial \mathscr{I}^{d_{\mathrm{I}}}}\left(\boldsymbol{s}^{d_{\mathrm{I}}} ; \mathscr{I}^{d_{\mathrm{I}}}\right)
$$

in terms of the stresses $\boldsymbol{s}^{d_{1}}$ (or, equivalently, $\boldsymbol{\sigma}^{d_{\mathrm{I}}}=\mathbb{P}^{d_{\mathrm{I}}^{\mathrm{T}}} \boldsymbol{s}^{d_{\mathrm{I}}}$ ) associated to the damage mechanism $d_{\mathrm{I}}$. The damage surfaces (2.25) can then be expressed as

$$
\hat{\phi}^{d_{1}}\left(\boldsymbol{s}^{d_{1}} ; \mathscr{I}^{d_{1}}\right):=\phi^{d_{1}}\left(\widehat{\mathscr{Q}^{d_{1}}}\left(\boldsymbol{s}^{d_{1}} ; \mathscr{I}^{d_{1}}\right)\right)
$$

in terms of the damage stresses $\boldsymbol{s}^{d_{1}}$ and the conjugate internal variables $\mathscr{I}^{d_{1}}$. The equations of the final plastic damage model are summarized in Table 1 . 


\section{Remark 2.4.}

1. A sufficient condition for the invertibility of the relation (2.37) is the convexity of the damage potentials $\widehat{W}^{d_{\mathrm{I}}}\left(\boldsymbol{e}^{d_{\mathrm{I}}} ; \mathscr{I}^{d_{\mathrm{I}}}\right)$ in the damage strain argument $\boldsymbol{e}^{d_{\mathrm{I}}}$ (that is, for fixed $\left.\mathscr{I}^{d_{\mathrm{I}}}\right)$, which also implies the convexity of the complementary functions $\chi^{d_{\mathrm{I}}}$ in the stresses $\boldsymbol{s}^{d_{\mathrm{I}}}$. This convexity applies for the cases of interest described in Section 3, and it is assumed hereafter, once the damage mechanism is activated.

2. The construction of the damage linear subspace $\mathscr{V}^{d_{1}}$ is, in fact, motivated by the final relation (2.43) defining the damage surfaces in terms of the specific damage stress components $\boldsymbol{s}^{d_{1}}$.

\subsection{The rate equations}

To identify better the damage response introduced by the considerations presented in the previous sections by the total stress-strain relations, we derive in this section the tangent rate equations associated to the general models developed above. As noted in Section 1, one of the main advantages of the proposed formulation is the modularity in the treatment of simultaneous damage mechanisms. We can exploit this modularity by first deriving the tangent rate form of the equations for each of the damage mechanisms to account for their combined action together with the elastoplastic response of the material afterwards. In this way, we consider a given damage mechanism $d_{\mathrm{I}}=1, n_{\mathrm{dam}}$ and derive the rate equation

$$
\dot{\boldsymbol{s}}^{d_{\mathrm{I}}}=\mathbf{c}_{l u}^{d_{1}} \dot{e}^{d_{\mathrm{I}}}
$$

or, in components,

$$
\dot{s}_{a}^{d_{\mathrm{I}}}=\sum_{b=1}^{n_{d_{\mathrm{I}}}} c_{l u_{a b}}^{d_{\mathrm{I}}} \dot{e}_{b}^{d_{\mathrm{I}}} \quad\left(a=1, n_{d_{\mathrm{I}}}\right)
$$

for a tangent matrix $\mathbf{c}_{l u}^{d_{1}} \in \mathbb{R}^{n_{d_{1}} \times n_{d_{1}}}$ to be found, accounting for the active damage loading or inactive damage unloading state of the damage mechanism $d_{\mathrm{I}}$. We note the use in Eq. (2.44a,b) of the reduced arrays in $\mathscr{V}^{d_{1}}$, thus avoiding in the following developments the singularities associated with the components of the strain and stress not involved in the damage of the material.

\subsubsection{The tangent damage rate relations}

The rate form of Eq. (2.39) reads

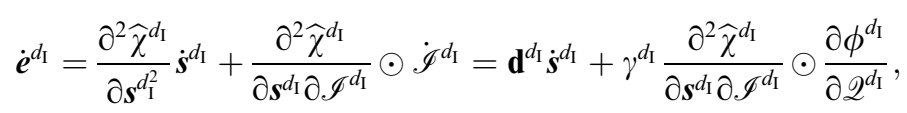

where we have used the evolution equation (2.26) and introduced the damage compliance $\mathbf{d}^{d_{\mathrm{I}}}$ through the definition

$$
\mathbf{d}^{d_{1}}:=\frac{\partial^{2} \widehat{\chi}^{d_{1}}}{\partial \boldsymbol{s}_{1}^{d_{1}^{2}}}
$$

The symmetry of the compliance $\mathbf{d}^{d_{\mathrm{I}}}$ follows. As noted in Remark 2.4, we assume the convexity of the damage potential $\widehat{\chi}\left(\boldsymbol{s}^{d_{\mathrm{I}}} ; \mathscr{I}^{d_{\mathrm{I}}}\right)$ in the stress component, hence resulting in the positive definiteness of the compliance $\mathbf{d}^{d_{\mathrm{I}}}$ in Eq. (2.46) (and thus invertible), once the damage mechanism $d_{\mathrm{I}}$ is activated. We introduce the notation

$$
\mathbf{c}^{d_{1}}:=\mathbf{d}^{d^{-1}}
$$

for the damage tangent. Similarly, the rate form of Eq. (2.42) leads to the relation 


$$
\begin{aligned}
\dot{\mathscr{Q}}^{d_{\mathrm{I}}} & =\frac{\partial^{2} \widehat{\chi}^{d_{\mathrm{I}}}}{\partial \mathscr{I}^{d_{\mathrm{I}}} \partial \boldsymbol{s}^{d_{\mathrm{I}}}} \dot{\boldsymbol{s}}^{d_{\mathrm{I}}}+\frac{\partial^{2} \widehat{\chi}^{d_{\mathrm{I}}}}{\partial \mathscr{I}^{d_{\mathrm{I}}^{2}}} \odot \dot{I}^{d_{\mathrm{I}}} \\
& =\frac{\partial^{2} \widehat{\chi}^{d_{\mathrm{I}}}}{\partial \mathscr{I}^{d_{\mathrm{I}}} \partial \boldsymbol{s}^{d_{\mathrm{I}}}} \dot{\boldsymbol{s}}^{d_{\mathrm{I}}}+\gamma^{d_{\mathrm{I}}} \frac{\partial^{2} \widehat{\chi}^{d_{\mathrm{I}}}}{\partial \mathscr{I}^{d_{\mathrm{I}}^{2}}} \odot \frac{\partial \phi^{d_{\mathrm{I}}}}{\partial \mathscr{Q}^{d_{\mathrm{I}}}} \\
\dot{\mathscr{Q}}^{d_{\mathrm{I}}} & =\frac{\partial^{2} \widehat{\chi}^{d_{\mathrm{I}}}}{\partial \mathscr{I}^{d_{\mathrm{I}}} \partial \boldsymbol{s}^{d_{\mathrm{I}}}} \mathbf{c}^{d_{\mathrm{I}}} \boldsymbol{e}^{d_{\mathrm{I}}}-\gamma^{d_{\mathrm{I}}}\left[\frac{\partial^{2} \widehat{\chi}^{d_{\mathrm{I}}}}{\partial \mathscr{I}^{d_{1}} \partial \boldsymbol{s}^{d_{\mathrm{I}}}} \mathbf{c}^{d_{\mathrm{I}}} \frac{\partial^{2} \widehat{\chi}^{d_{\mathrm{I}}}}{\partial \boldsymbol{s}^{d_{\mathrm{I}}} \partial \mathscr{I}^{d_{\mathrm{I}}}}-\frac{\partial^{2} \widehat{\chi}^{d_{\mathrm{I}}}}{\partial \mathscr{I}^{d_{\mathrm{I}}^{2}}}\right] \odot \frac{\partial \phi^{d_{\mathrm{I}}}}{\partial \mathscr{V}^{d_{\mathrm{I}}}}
\end{aligned}
$$

after using Eqs. (2.45) and (2.47). The damage multiplier $\gamma^{d_{\mathrm{I}}}$ is obtained by imposing damage consistency during persistent damage, as follows.

(i) Damaged unloading. No further damage of the material occurs in this case, with

$$
\phi^{d_{1}}<0 \Rightarrow \gamma^{d_{1}}=0
$$

by the Kuhn-Tucker condition (2.27c). Therefore, Eq. (2.45) leads to

$$
\dot{\boldsymbol{s}}^{d_{\mathrm{I}}}=\mathbf{c}^{d_{\mathrm{I}}} \dot{\boldsymbol{e}}^{d_{\mathrm{I}}} \Rightarrow \mathbf{c}_{l u}^{d_{\mathrm{I}}}=\mathbf{c}^{d_{\mathrm{I}}} \quad \text { for (damaged) unloading }
$$

with no further evolution of the compliance associated to the damage mechanism in this case.

(ii) Damage loading. The imposition of the consistency condition (2.28)

$$
\dot{\phi}^{d_{\mathrm{I}}}=\frac{\partial \phi^{d_{\mathrm{I}}}}{\partial \mathscr{Q}^{d_{\mathrm{I}}}} \odot \dot{\mathscr{2}}^{d_{\mathrm{I}}}=0
$$

leads in combination with Eq. (2.49) to

$$
\gamma^{d_{\mathrm{I}}}=\frac{1}{\Delta^{d_{\mathrm{I}}}}\left(\frac{\partial \phi^{d_{\mathrm{I}}}}{\partial \mathscr{Q}^{d_{\mathrm{I}}}} \odot \frac{\partial^{2} \widehat{\chi}^{d_{\mathrm{I}}}}{\partial \mathscr{I}^{d_{\mathrm{I}}} \partial \boldsymbol{s}^{d_{\mathrm{I}}}}\right) \mathbf{c}^{d_{\mathrm{I}}} \dot{\boldsymbol{e}}^{d_{\mathrm{I}}}
$$

where

$$
\Delta^{d_{\mathrm{I}}}:=\frac{\partial \phi^{d_{\mathrm{I}}}}{\partial \mathscr{Q}^{d_{\mathrm{I}}}} \odot\left[\frac{\partial^{2} \widehat{\chi}^{d_{\mathrm{I}}}}{\partial \mathscr{I}^{d_{\mathrm{I}}} \partial \boldsymbol{s}^{d_{\mathrm{I}}}} \mathbf{c}^{d_{\mathrm{I}}} \frac{\partial^{2} \widehat{\chi}^{d_{\mathrm{I}}}}{\partial \boldsymbol{s}^{d_{\mathrm{I}}} \partial \mathscr{I}^{d_{\mathrm{I}}}}-\frac{\partial^{2} \widehat{\chi}^{d_{\mathrm{I}}}}{\partial \mathscr{I}^{d_{\mathrm{I}}^{2}}}\right] \odot \frac{\partial \phi^{d_{\mathrm{I}}}}{\partial \mathscr{Q}^{d_{\mathrm{I}}}}
$$

which is assumed to be positive $\Delta^{d_{\mathrm{I}}}>0$; see Remark 2.5 below. The introduction of this expression of $\gamma^{d_{\mathrm{I}}}$ in Eq. (2.45) leads to the tangent rate Eq. $(2.44 a, b)$ with

$$
\mathbf{c}_{l u}^{d_{\mathrm{I}}}=\mathbf{c}^{d_{\mathrm{I}}}-\frac{1}{\Delta^{d_{\mathrm{I}}}}\left[\mathbf{c}^{d_{\mathrm{I}}}\left(\frac{\partial^{2} \widehat{\chi}^{d_{\mathrm{I}}}}{\partial \boldsymbol{s}^{d_{1}} \partial \mathscr{I}^{d_{\mathrm{I}}}} \odot \frac{\partial \phi^{d_{\mathrm{I}}}}{\partial \mathscr{Q}^{d_{\mathrm{I}}}}\right)\right] \otimes\left[\mathbf{c}^{d_{\mathrm{I}}}\left(\frac{\partial^{2} \widehat{\chi}^{d_{\mathrm{I}}}}{\partial \boldsymbol{s}^{d_{\mathrm{I}}} \partial \mathscr{I}^{d_{\mathrm{I}}}} \odot \frac{\partial \phi^{d_{\mathrm{I}}}}{\partial \mathscr{Q}^{d_{\mathrm{I}}}}\right)\right] \text {. }
$$

The symmetry of this tangent is to be noted.

Remark 2.5. The constitutive assumption $\Delta^{d_{1}}>0$ in Eq. (2.54) is to be verified on a case by case basis in the model examples described in Section 3. With this assumption, Eq. (2.53) leads to the equivalent condition for persistent damage loading

$$
\left(\frac{\partial \phi^{d_{\mathrm{I}}}}{\partial \mathscr{Q}^{d_{\mathrm{I}}}} \odot \frac{\partial^{2} \widehat{\chi}^{d_{\mathrm{I}}}}{\partial \mathscr{I}^{d_{\mathrm{I}}} \partial \boldsymbol{s}^{d_{\mathrm{I}}}}\right) \mathbf{c}^{d_{1}} \boldsymbol{e}^{d_{\mathrm{I}}}>0
$$

for the damage mechanism $d_{\mathrm{I}}$, after noting the constraint $\gamma^{d_{1}}>0$ in the first term of Eq. (2.27).

\subsubsection{The global elastoplastic-damage tangent}

The above considerations focused on a given damage mechanism $d_{\mathrm{I}}$. Global relations between the rates of total strain $\dot{\boldsymbol{\varepsilon}}$ and stress $\dot{\boldsymbol{\sigma}}$ can be obtained as follows. The rate form of the elastic relation (2.12) leads to 


$$
\dot{\sigma}=\mathbf{C}^{\mathrm{e}} \dot{\boldsymbol{\varepsilon}}^{\mathrm{e}}=\mathbf{C}^{\mathrm{e}}\left(\dot{\boldsymbol{\varepsilon}}-\dot{\boldsymbol{\varepsilon}}^{\mathrm{d}}-\dot{\boldsymbol{\varepsilon}}^{\mathrm{p}}\right) \quad \text { for } \mathbf{C}^{\mathrm{e}}:=\frac{\partial^{2} W^{\mathrm{e}}}{\partial \boldsymbol{\varepsilon}^{\mathrm{e}^{2}}},
$$

the symmetric elastic tangent (not necessarily constant). The plastic strain rates $\dot{\boldsymbol{\varepsilon}}^{\mathrm{p}}$ in this last expression are eliminated in the usual way. Indeed, the imposition of the plastic consistency (2.31) in combination with Eq. (2.57) leads to the expression of the plastic multiplier

$$
\gamma^{\mathrm{p}}=\frac{1}{\Delta^{\mathrm{p}}} \boldsymbol{n}_{\phi^{\mathrm{p}}}: \mathbf{C}^{\mathrm{e}}\left(\dot{\boldsymbol{\varepsilon}}-\dot{\boldsymbol{\varepsilon}}^{\mathrm{d}}\right)
$$

where we have introduced the notation

$$
\boldsymbol{n}_{\phi^{\mathrm{p}}}:=\frac{\partial \phi^{\mathrm{p}}}{\partial \boldsymbol{\sigma}} \quad \text { and } \quad \Delta^{\mathrm{p}}:=\boldsymbol{n}_{\phi^{\mathrm{p}}}: \mathbf{C}^{\mathrm{e}} \boldsymbol{n}_{\phi^{\mathrm{p}}}+\left(\frac{\partial \phi^{\mathrm{p}}}{\partial \mathscr{Q}^{\mathrm{p}}}\right)^{2} K^{\mathrm{p}}
$$

for the hardening modulus $K^{\mathrm{p}}:=\partial^{2} \mathscr{H}^{\mathrm{p}} / \partial \alpha^{\mathrm{p}^{2}}$ in terms of the plastic hardening potential $\mathscr{H}^{\mathrm{p}}$. Combining Eqs. (2.29a), (2.57) and (2.58), we arrive at the tangent relation

$$
\dot{\boldsymbol{\sigma}}=\mathbf{C}^{\mathrm{ep}}\left(\dot{\boldsymbol{\varepsilon}}-\dot{\boldsymbol{\varepsilon}}^{\mathrm{d}}\right)
$$

for the elastoplastic tangent $\mathbf{C}^{\mathrm{ep}}$

$$
\mathbf{C}^{\mathrm{ep}}=\mathbf{C}^{\mathrm{e}}-\frac{1}{\Delta^{\mathrm{p}}} \mathbf{C}^{\mathrm{e}} \boldsymbol{n}_{\phi^{\mathrm{p}}} \otimes \mathbf{C}^{\mathrm{e}} \boldsymbol{n}_{\phi^{\mathrm{p}}}
$$

a symmetric tangent in the assumed associated case.

The combination of Eqs. (2.19b) and (2.60) leads to

$$
\dot{\boldsymbol{s}}^{d_{\mathrm{I}}}=\mathbb{P}^{d_{\mathrm{I}}}: \dot{\boldsymbol{\sigma}}=\mathbb{P}^{d_{\mathrm{I}}}: \mathbf{C}^{\mathrm{ep}}\left(\dot{\boldsymbol{\varepsilon}}-\sum_{d_{J}=1}^{n_{\mathrm{dam}}} \mathbb{P}^{d_{J}^{\mathrm{T}}} \dot{\boldsymbol{e}}^{d_{J}}\right) \quad \text { for } d_{\mathrm{I}}=1, n_{\mathrm{dam}},
$$

which with (2.44a) leads to

$$
\sum_{d_{J}=1}^{n_{\mathrm{dam}}}\left[\mathbf{c}_{l u}^{d_{\mathrm{I}}} \delta_{d_{\mathrm{I}} d_{J}}+\mathbb{P}^{d_{\mathrm{I}}}: \mathbf{C}^{\mathrm{ep}} \mathbb{P}^{d_{J}^{\mathrm{T}}} \mathbf{c}_{l u}^{d_{J}}\right] \dot{\boldsymbol{e}}^{d_{J}}=\mathbb{P}^{d_{\mathrm{I}}}: \mathbf{C}^{\mathrm{ep}} \dot{\boldsymbol{\varepsilon}} \quad \text { for } d_{\mathrm{I}}=1, n_{\mathrm{dam}} .
$$

Following the notation introduced in Eq. (2.8), we consider the matrix

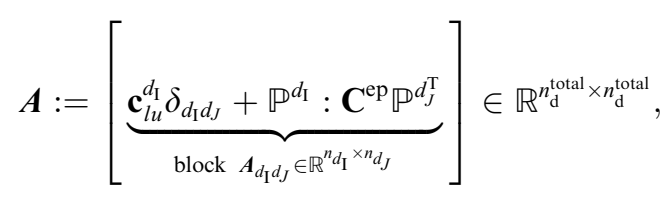

where

$$
n_{\mathrm{d}}^{\text {total }}=\sum_{d_{\mathrm{I}}=1}^{n_{\mathrm{dam}}} n_{d_{\mathrm{I}}}
$$

the total number of damage variables. The symmetry of the matrix $\boldsymbol{A}$ is to be noted. From Eq. (2.63), we conclude that

$$
\dot{\boldsymbol{e}}^{d_{\mathrm{I}}}=\sum_{d_{J}=1}^{n_{\mathrm{dam}}}\left(\boldsymbol{A}^{-1}\right)_{d_{\mathrm{I}} d_{J}} \mathbb{P}^{d_{J}}: \mathbf{C}^{\mathrm{ep}} \dot{\boldsymbol{\varepsilon}}
$$

where $\left(\boldsymbol{A}^{-1}\right)_{d_{1} d_{J}} \in \mathbb{R}^{n_{d_{1}} \times n_{d_{J}}}$ denotes the block of the inverse of matrix $\boldsymbol{A}$ associated to the mechanisms $d_{\mathrm{I}}$ and $d_{J}$. 
The final tangent rate equation is obtained as

$$
\begin{aligned}
& \dot{\boldsymbol{\sigma}}=\mathbf{C}^{\mathrm{ep}}\left(\dot{\boldsymbol{\varepsilon}}-\sum_{d_{\mathrm{I}}=1}^{n_{\mathrm{dam}}} \mathbb{P}^{d_{\mathrm{I}}^{\mathrm{T}}} \dot{\boldsymbol{e}}^{d_{\mathrm{I}}}\right) \\
& \dot{\boldsymbol{\sigma}}=\left[\mathbf{C}^{\mathrm{ep}}-\sum_{d_{\mathrm{I}}, d_{J}=1}^{n_{\mathrm{dam}}} \mathbf{C}^{\mathrm{ep}}: \mathbb{P}^{d_{\mathrm{I}}^{\mathrm{T}}}\left(\boldsymbol{A}^{-1}\right)_{d_{\mathrm{I}} d_{J}} \mathbb{P}^{d_{J}}: \mathbf{C}^{\mathrm{ep}}\right] \dot{\boldsymbol{\varepsilon}},
\end{aligned}
$$

identifying the final rate equation

$$
\dot{\boldsymbol{\sigma}}=\mathbf{C}^{\mathrm{epd}} \dot{\boldsymbol{\varepsilon}}, \quad \text { where } \mathbf{C}^{\mathrm{epd}}=\mathbf{C}^{\mathrm{ep}}-\sum_{d_{\mathrm{I}}, d_{J}=1}^{n_{\mathrm{dam}}} \mathbf{C}^{\mathrm{ep}}: \mathbb{P}^{d_{\mathrm{I}}^{\mathrm{T}}}\left(\boldsymbol{A}^{-1}\right)_{d_{\mathrm{I}} d_{J}} \mathbb{P}^{d_{J}}: \mathbf{C}^{\mathrm{ep}} .
$$

We note the symmetry of the final tangent. The damage incorporated in the tangent response of the material is apparent in the final relation (2.69) through the evolution of the tangent stiffness of the material from its elastoplastic value $\mathbf{C}^{\mathrm{ep}}$. The degradation of this tangent response is accomplished in the proposed formulation through a direct modeling of the physical damage mechanisms. To illustrate better the added compliance added to the global tangent response of the material by these mechanisms, we present next an alternative derivation of the tangent in terms of the damage compliances.

\subsubsection{Alternative form of the damage tangent}

An alternative form of the tangent (2.69) can be obtained by deriving first the corresponding compliance, when possible. We first note that the compliance $\mathbf{d}_{l u}^{d_{1}}=\mathbf{c}_{l u}^{d_{1}^{-1}}$ associated to the tangent $\mathbf{c}_{l u}^{d_{1}}$ in Eq. (2.55) for the damage mechanism $d_{\mathrm{I}}$ can be obtained using the Sherman-Morrison formula (Golub and van Loan, 1989, page 51), leading to the expression

$$
\mathbf{d}_{l u}^{d_{\mathrm{I}}}=\mathbf{d}^{d_{\mathrm{I}}}+\frac{1}{\tilde{\Delta}^{d_{\mathrm{I}}}}\left(\frac{\partial^{2} \widehat{\chi}^{d_{\mathrm{I}}}}{\partial \boldsymbol{s}^{d_{\mathrm{I}}} \partial \mathscr{I}^{d_{\mathrm{I}}}} \odot \frac{\partial \phi^{d_{\mathrm{I}}}}{\partial \mathscr{Q}^{d_{\mathrm{I}}}}\right) \otimes\left(\frac{\partial^{2} \widehat{\chi}^{d_{\mathrm{I}}}}{\partial \boldsymbol{s}^{d_{\mathrm{I}}} \partial \mathscr{I}^{d_{\mathrm{I}}}} \odot \frac{\partial \phi^{d_{\mathrm{I}}}}{\partial \mathscr{Q}^{d_{\mathrm{I}}}}\right)
$$

where

$$
\begin{aligned}
\tilde{\Delta}^{d_{\mathrm{I}}} & =\Delta^{d_{\mathrm{I}}}-\frac{\partial \phi^{d_{\mathrm{I}}}}{\partial \mathscr{Q}^{d_{\mathrm{I}}}} \odot\left[\frac{\partial^{2} \widehat{\chi}^{d_{\mathrm{I}}}}{\partial \mathscr{I}^{d_{\mathrm{I}}} \partial \boldsymbol{s}^{d_{\mathrm{I}}}} \mathbf{c}^{d_{\mathrm{I}}} \frac{\partial^{2} \widehat{\chi}^{d_{\mathrm{I}}}}{\partial \boldsymbol{s}^{d_{\mathrm{I}}} \partial \mathscr{I}^{d_{\mathrm{I}}}}\right] \odot \frac{\partial \phi^{d_{\mathrm{I}}}}{\partial \mathscr{Q}^{d_{\mathrm{I}}}} \\
& =-\frac{\partial \phi^{d_{\mathrm{I}}}}{\partial \mathscr{2}^{d_{\mathrm{I}}}} \odot \frac{\partial^{2} \widehat{\chi}^{d_{\mathrm{I}}}}{\partial \mathscr{I}^{d_{\mathrm{I}}^{2}}} \odot \frac{\partial \phi^{d_{\mathrm{I}}}}{\partial \mathscr{2}^{d_{\mathrm{I}}}}
\end{aligned}
$$

assuming $\tilde{\Delta}^{d_{\mathrm{I}}} \neq 0$; see Remark 2.6. Expression (2.70) can be obtained alternatively by combining Eq. (2.48) (instead of Eq. (2.49)) and the consistency condition (2.52). For the case of no further damage (i.e. damaged unloading) characterized by the tangent relation (2.51), the corresponding compliance is simply obtained as

$$
\mathbf{d}_{l u}^{d_{\mathrm{I}}}=\mathbf{d}^{d_{\mathrm{I}}}
$$

given by Eq. (2.46).

The global rate equation is then easily obtained from the inverse of the elastic rate Eq. (2.67) in combination with the inverse of the rate equation (2.44a), that is,

$$
\mathbf{D}^{\mathrm{ep}} \dot{\boldsymbol{\sigma}}=\dot{\boldsymbol{\varepsilon}}-\sum_{d_{\mathrm{I}}=1}^{n_{\mathrm{dam}}}(\underbrace{\sum_{a=1}^{n_{d_{\mathrm{I}}}} \mathbb{P}_{a}^{d_{\mathrm{I}}} \dot{e}_{a}^{d_{\mathrm{I}}}}_{\dot{\boldsymbol{\varepsilon}}^{d_{\mathrm{I}}}}) \text { and } \dot{e}_{a}^{d_{\mathrm{I}}}=\sum_{b=1}^{n_{d_{\mathrm{I}}}} \mathbf{d}_{l u_{a b}}^{d_{\mathrm{I}}} \underbrace{\mathbb{P}_{a}^{d_{\mathrm{I}}}: \dot{\boldsymbol{\sigma}}}_{\dot{s}_{a}^{d_{\mathrm{I}}}}
$$


for the elastoplastic compliance $\mathbf{D}^{\mathrm{ep}}:=\mathbf{C}^{\mathrm{e}^{-1}}$, thus leading to the elastoplastic-damage compliance

$$
\dot{\boldsymbol{\varepsilon}}=\mathbf{D}^{\text {epd }} \dot{\boldsymbol{\sigma}}, \quad \text { where } \mathbf{D}^{\text {epd }}=\mathbf{D}^{\mathrm{ep}}+\sum_{d_{\mathrm{I}}=1}^{n_{\mathrm{dam}}} \mathbf{D}_{l u}^{d_{\mathrm{I}}}
$$

with the damage compliance contribution

$$
\mathbf{D}_{l u}^{d_{\mathrm{I}}}=\sum_{a, b=1}^{n_{d_{\mathrm{I}}}} \mathbf{d}_{l u_{a b}}^{d_{\mathrm{I}}} \mathbb{P}_{a}^{d_{\mathrm{I}}} \otimes \mathbb{P}_{b}^{d_{\mathrm{I}}}
$$

for the damage mechanism $d_{\mathrm{I}}$. The final expression (2.74) identifies clearly the added compliance to the tangent material response

Remark 2.6. The compliance (2.70) is only well defined when the elastoplastic tangent $\boldsymbol{C}^{\mathrm{ep}}$ is invertible and $\tilde{\Delta}^{d_{1}} \neq 0$ in (2.71) (otherwise the tangent $\boldsymbol{c}_{l u}^{d_{\mathrm{I}}}$ in Eq. (2.55) is singular). After observing the nature of $\tilde{\Delta}^{d_{\mathrm{I}}}$ in Eq. (2.71), we note that this situation may occur in the case of perfect damage (no hardening/softening response of the material) as it is shown in the model examples considered in Section 3. The same considerations apply to the invertibility of the elastoplastic tangent given by Eq. (2.61). For these reasons, the form of the tangent (2.69), not requiring this inversion before adding the elastic contributions, is to be preferred in the numerical implementation.

\section{Model examples}

We consider in this section a generic elastoplastic-damage model involving a quadratic damage potential, thus introducing a secant damage compliance. Secant damage compliance have been considered in Ortiz (1985), leading to similar developments to the ones presented herein for the purely elastic-damage case only. These considerations lead to a linear stress-strain relation in unloading and reloading when combined with a quadratic elastic potential, as illustrated in Fig. 2 in the context of a uniaxial tension test.

After describing this generic quadratic damage model in Section 3.1 in the general elastoplastic-damage framework developed in Section 2, we consider in Section 3.2 several existing models, developing their formulation within the proposed framework. A single damage mechanism (i.e. $n_{\mathrm{dam}}=1$ ) is considered in Section 3, for simplicity in the presentation, but each and every particular case considered next fits in the general framework presented in Section 2. The several possible combinations of these different cases allow the formulation of more general models. In this way, we may consider from simple extensions like smeared crack models based on several crack orientations to more involved cases like a smeared crack model combined with a scalar isotropic damage model to mention two possible examples.

\subsection{A generic quadratic damage model}

We consider a damage mechanism characterized by the generic quadratic potential

$$
\widehat{W}^{\mathrm{d}}\left(\boldsymbol{e}^{\mathrm{d}} ; \mathscr{I}^{\mathrm{d}}\right)=\frac{1}{2} \boldsymbol{e}^{\mathrm{d}}: \mathbf{c}^{\mathrm{d}} \boldsymbol{e}^{\mathrm{d}}+\mathscr{H}^{\mathrm{d}}\left(\alpha^{\mathrm{d}}\right)
$$

with the internal variables

$$
\mathscr{I}^{\mathrm{d}}=\left\{\mathbf{d}^{\mathrm{d}}, \alpha^{\mathrm{d}}\right\} \quad \text { for } \mathbf{d}^{\mathrm{d}}:=\mathbf{c}^{\mathrm{d}^{-1}}
$$

and for the scalar variable $\alpha^{\mathrm{d}}$. The generic function $\mathscr{H}^{\mathrm{d}}\left(\alpha^{\mathrm{d}}\right)$ accounts for an isotropic hardening/softening damage law of the material. The damage space $\mathscr{V}^{\mathrm{d}}$ defined in terms of the projection matrices $\mathbb{P}^{\mathrm{d}}$ by 
Eq. (2.4), so the damage strains are $\boldsymbol{\varepsilon}^{\mathrm{d}}=\mathbb{P}^{\mathrm{d}^{\mathrm{T}}} \boldsymbol{e}^{\mathrm{d}}$, is to be specified for a particular damage surface; see examples below. We also write the global compliance

$$
\mathbf{D}^{\mathrm{d}}=\sum_{a, b=1}^{n_{\mathrm{d}}} \mathbf{d}_{a b}^{\mathrm{d}} \mathbb{P}_{a}^{\mathrm{d}} \otimes \mathbb{P}_{b}^{\mathrm{d}}
$$

and the global tangent

$$
\mathbf{C}^{\mathrm{d}}=\sum_{a, b=1}^{n_{d_{\mathrm{I}}}} \mathbf{c}_{a b}^{\mathrm{d}} \mathbb{P}_{a}^{\mathrm{d}} \otimes \mathbb{P}_{b}^{\mathrm{d}} \quad \text { for } \mathbf{c}_{a b}^{\mathrm{d}}:=\mathbb{P}_{a}^{\mathrm{d}}: \mathbf{C}^{\mathrm{d}} \mathbb{P}_{b}^{\mathrm{d}}
$$

leading to the equality

$$
\boldsymbol{\varepsilon}^{\mathrm{d}}: \mathbf{C}^{\mathrm{d}} \boldsymbol{\varepsilon}^{\mathrm{d}}=\boldsymbol{e}^{d_{\mathrm{I}}} \cdot \mathbf{c}^{\mathrm{d}} \boldsymbol{e}^{d_{\mathrm{I}}}
$$

for the quadratic term in Eq. (3.1). We write $\mathbf{C}^{\mathrm{d}^{-1}}=\mathbf{D}^{\mathrm{d}}$ in $\mathscr{V}^{\mathrm{d}}$, i.e., the generalized inverse.

The complementary energy associated to $\widehat{W}^{\mathrm{d}}$ in Eq. (3.1) is given by

$$
\begin{aligned}
\widehat{\chi}^{\mathrm{d}}\left(\boldsymbol{s}^{\mathrm{d}} ; \mathbf{d}^{\mathrm{d}}, \alpha^{\mathrm{d}}\right) & =\frac{1}{2} \boldsymbol{s}^{\mathrm{d}}: \mathbf{d}^{\mathrm{d}} \boldsymbol{s}^{\mathrm{d}}-\mathscr{H}^{\mathrm{d}}\left(\alpha^{d}\right) \\
& =\frac{1}{2}\left(\boldsymbol{s}^{\mathrm{d}} \otimes \boldsymbol{s}^{\mathrm{d}}\right): \mathbf{d}^{\mathrm{d}}-\mathscr{H}^{\mathrm{d}}\left(\alpha^{\mathrm{d}}\right) \\
& =\frac{1}{2}\left(\boldsymbol{\sigma}^{\mathrm{d}} \otimes \boldsymbol{\sigma}^{\mathrm{d}}\right): \mathbf{D}^{\mathrm{d}}-\mathscr{H}^{d}\left(\alpha^{\mathrm{d}}\right)
\end{aligned}
$$

for $\boldsymbol{\sigma}^{\mathrm{d}}=\mathbb{P}^{\mathrm{d}^{\mathrm{T}}} \boldsymbol{s}^{\mathrm{d}}$, as a calculation based on Eq. (2.35) shows. The constitutive relations (2.40) and (2.42) read in this case

$$
\begin{aligned}
& \boldsymbol{e}^{\mathrm{d}}=\frac{\partial \widehat{\chi}^{\mathrm{d}}}{\partial \boldsymbol{s}^{\mathrm{d}}}=\mathbf{d}^{\mathrm{d}} \boldsymbol{s}^{\mathrm{d}} \quad\left(\text { or, equivalently, } \boldsymbol{\varepsilon}^{\mathrm{d}}=\mathbf{D}^{\mathrm{d}} \boldsymbol{\sigma}^{\mathrm{d}}=\mathbf{D}^{\mathrm{d}} \boldsymbol{\sigma}\right), \\
& \mathscr{Q}_{1}^{\mathrm{d}}=\frac{\partial \widehat{\chi}^{\mathrm{d}}}{\partial \mathbf{d}^{\mathrm{d}}}=\frac{1}{2}\left(\boldsymbol{s}^{\mathrm{d}} \otimes \boldsymbol{s}^{\mathrm{d}}\right), \\
& \mathscr{2}_{2}^{\mathrm{d}}=\frac{\partial \widehat{\chi}^{\mathrm{d}}}{\partial \alpha^{\mathrm{d}}}=-\frac{\partial \mathscr{H}^{\mathrm{d}}}{\partial \alpha^{\mathrm{d}}}=: q^{\mathrm{d}}
\end{aligned}
$$

leading to the expression (2.15) of the damage dissipation

$$
\mathscr{D}^{\mathrm{d}}=\frac{1}{2}\left(\boldsymbol{s}^{\mathrm{d}} \otimes \boldsymbol{s}^{\mathrm{d}}\right): \dot{\mathbf{d}}^{\mathrm{d}}+q^{\mathrm{d}} \dot{\alpha}^{\mathrm{d}} \geqslant 0 .
$$

Appropriate damage surfaces are defined then in this case as

$$
\phi^{\mathrm{d}}\left(\frac{1}{2}\left(\boldsymbol{s}^{\mathrm{d}} \otimes \boldsymbol{s}^{\mathrm{d}}\right), q^{\mathrm{d}}\right) \leqslant 0
$$

leading to the associated damage evolution equations

$$
\begin{aligned}
& \dot{\mathbf{d}}^{\mathrm{d}}=\gamma^{\mathrm{d}} \boldsymbol{N}_{\phi^{\mathrm{d}}} \quad \text { for } \boldsymbol{N}_{\phi^{\mathrm{d}}}:=\frac{\partial \phi^{\mathrm{d}}}{\partial\left(\frac{1}{2}\left(\boldsymbol{s}^{\mathrm{d}} \otimes \boldsymbol{s}^{\mathrm{d}}\right)\right)}, \\
& \dot{\alpha}^{\mathrm{d}}=\gamma^{\mathrm{d}} \frac{\partial \phi^{\mathrm{d}}}{\partial q^{\mathrm{d}}}
\end{aligned}
$$

with the Kuhn-Tucker loading/unloading conditions (2.27) and the consistency conditions (2.28).

Remark 3.1. The constraint (3.11) on the damage dissipation $\mathscr{D}^{\mathrm{d}}$ is automatically satisfied assuming a damage surface of the form 


$$
\phi^{\mathrm{d}}\left(\frac{1}{2} \boldsymbol{s}^{\mathrm{d}} \otimes \boldsymbol{s}^{\mathrm{d}}, q\right)=g^{\mathrm{d}}(\underbrace{\frac{1}{2} \boldsymbol{s}^{\mathrm{d}} \otimes \boldsymbol{s}^{\mathrm{d}}}_{2_{1}^{\mathrm{d}}})-(\underbrace{\omega_{0}^{\mathrm{d}}-q^{\mathrm{d}}\left(\alpha^{\mathrm{d}}\right)}_{\omega^{\mathrm{d}}\left(\alpha^{\mathrm{d}}\right) \geqslant 0}) \leqslant 0
$$

for a material parameter $\omega^{\mathrm{d}}(0)=\omega_{0}^{\mathrm{d}}>0$ (i.e. $\left.q^{\mathrm{d}}(0)=0\right)$ and a function $g^{\mathrm{d}}(\cdot)$ positively homogeneous of degree 1 . We recall that a positively homogeneous function of degree $m \geqslant 1$ is defined by

$$
g^{\mathrm{d}}\left(\lambda \mathscr{2}_{1}^{\mathrm{d}}\right)=\lambda^{m} g^{\mathrm{d}}\left(\mathscr{2}_{1}^{\mathrm{d}}\right) \quad \forall \lambda \in \mathbb{R}^{+} \Rightarrow \frac{\partial g^{\mathrm{d}}}{\partial \mathscr{Q}_{1}^{\mathrm{d}}}: \mathscr{Q}_{1}^{\mathrm{d}}=m g^{\mathrm{d}},
$$

the Euler's theorem of homogeneous functions. In the case of interest $(m=1)$, we obtain after some straightforward algebraic manipulations

$$
\mathscr{D}^{\mathrm{d}}=\frac{1}{2}\left(\boldsymbol{s}^{\mathrm{d}} \otimes \boldsymbol{s}^{\mathrm{d}}\right): \dot{\mathbf{d}}^{\mathrm{d}}+q^{\mathrm{d}} \dot{\alpha}^{\mathrm{d}}=\gamma^{\mathrm{d}} \omega_{0}^{\mathrm{d}} \geqslant 0
$$

given the Kuhn-Tucker condition (2.27).

\subsubsection{An alternative characterization of the damage}

The damage evolution equations (3.13)-(3.14) have been obtained, following the general framework presented in Section 2, in terms of the damage surface (3.12) function of the fourth-order tensor $2_{1}^{\mathrm{d}}=\frac{1}{2}\left(\boldsymbol{s}^{\mathrm{d}} \otimes \boldsymbol{s}^{\mathrm{d}}\right)$. It is common, however, to characterize the damage of the material in terms of a damage surface of the form

$$
\tilde{\phi}^{\mathrm{d}}\left(\boldsymbol{s}^{\mathrm{d}}, q^{\mathrm{d}}\right) \leqslant 0
$$

in terms of the stress tensor $\boldsymbol{s}^{\mathrm{d}}$. The damage evolution equations for this case can still be obtained using the principle of maximum damage dissipation as follows.

Consider the Lagrangian associated to the dissipation functional (3.11) and the constraint introduced by the damage surface (3.18), that is,

$$
\mathscr{L}^{\mathrm{d}}\left(\boldsymbol{s}^{\mathrm{d}}, q^{\mathrm{d}}, \gamma^{\mathrm{d}} ; \dot{\boldsymbol{e}}^{\mathrm{d}}, \dot{\alpha}^{\mathrm{d}}\right):=\frac{1}{2} \boldsymbol{s}^{\mathrm{d}} \cdot \dot{\mathbf{d}}^{\mathrm{d}} \boldsymbol{s}^{\mathrm{d}}+q^{\mathrm{d}} \dot{\alpha}^{\mathrm{d}}-\gamma^{\mathrm{d}} \tilde{\phi}^{\mathrm{d}}\left(\tilde{\boldsymbol{s}}^{\mathrm{d}}, q^{\mathrm{d}}\right)
$$

for a Lagrange multiplier $\gamma^{\mathrm{d}} \geqslant 0$ imposing the unilateral constraint $\tilde{\phi}^{\mathrm{d}} \leqslant 0$. The stationarity of the Lagrangian (3.19) for fixed rates $\dot{\mathbf{d}}^{\mathrm{d}}$ and $\dot{\alpha}^{\mathrm{d}}$ of the damage variables leads to the evolution equations

$$
\begin{aligned}
& \dot{\mathbf{d}}^{\mathrm{d}} \boldsymbol{s}^{\mathrm{d}}=\gamma^{\mathrm{d}} \boldsymbol{n}_{\tilde{\phi}^{\mathrm{d}}}, \\
& \dot{\alpha}^{\mathrm{d}}=\gamma^{\mathrm{d}} \frac{\partial \tilde{\phi}^{\mathrm{d}}}{\partial q^{\mathrm{d}}}
\end{aligned}
$$

with the Kuhn-Tucker complementary conditions (2.27), where we have introduced the notation

$$
\boldsymbol{n}_{\tilde{\phi}^{\mathrm{d}}}:=\frac{\partial \tilde{\phi}^{\mathrm{d}}}{\partial \boldsymbol{s}^{\mathrm{d}}} .
$$

Clearly, the only component of the compliance rate affecting the dissipation functional (3.19) is the compliance along the direction defined by the current damage stress $\boldsymbol{s}^{\mathrm{d}}$. Hence, the relation (3.20) shows that the principle of maximum dissipation in combination of a damage surface in terms of the stress $\boldsymbol{s}^{\mathrm{d}}$ alone determines only this component of the compliance, leaving arbitrary the component in the direction orthogonal (in stress space) to the current stress. A particular evolution equation for the compliance $\mathbf{d}^{\mathrm{d}}$ satisfying the relation (3.20a) is given by

$$
\dot{\mathbf{d}}^{\mathrm{d}}=\gamma^{\mathrm{d}} \frac{\boldsymbol{n}_{\tilde{\phi}^{\mathrm{d}}} \otimes \boldsymbol{n}_{\tilde{\phi}^{\mathrm{d}}}}{\boldsymbol{n}_{\tilde{\phi}^{\mathrm{d}}} \cdot \boldsymbol{s}^{\mathrm{d}}}
$$


as long as $\boldsymbol{n}_{\tilde{\phi}^{\mathrm{d}}} \cdot \boldsymbol{s}^{\mathrm{d}} \neq 0$; see Remark 3.2 (2). Evolution equations of the form given by Eq. (3.22) can be found in the works of Simo et al. (1993) and Govindjee et al. (1995) in the context of damage models based on the evolution of the a total compliance.

The two different expressions (3.13) and (3.22) can be reconciled by noting that the consideration of the function

$$
\tilde{\phi}^{\mathrm{d}}\left(\boldsymbol{s}^{\mathrm{d}}, q^{\mathrm{d}}\right):=\phi^{\mathrm{d}}\left(\frac{1}{2}\left(\boldsymbol{s}^{\mathrm{d}} \otimes \boldsymbol{s}^{\mathrm{d}}\right), q^{\mathrm{d}}\right)
$$

for a given function $\phi^{\mathrm{d}}(\cdot)$, leads to the differential relation

$$
\begin{aligned}
\mathrm{d} \tilde{\phi}^{\mathrm{d}} & =\boldsymbol{n}_{\tilde{\phi}^{\mathrm{d}}} \cdot \mathrm{d} \boldsymbol{s}^{\mathrm{d}}+\frac{\partial \tilde{\phi}^{\mathrm{d}}}{\partial q^{\mathrm{d}}} \mathrm{d} q^{\mathrm{d}} \\
& =\frac{\boldsymbol{n}_{\tilde{\phi}^{\mathrm{d}}} \otimes \boldsymbol{n}_{\tilde{\phi}^{\mathrm{d}}}}{\boldsymbol{n}_{\tilde{\phi}^{\mathrm{d}}} \cdot \boldsymbol{s}^{\mathrm{d}}}: \underbrace{\frac{1}{2}\left[\mathrm{~d} \boldsymbol{s}^{\mathrm{d}} \otimes \boldsymbol{s}^{\mathrm{d}}+\boldsymbol{s}^{\mathrm{d}} \otimes \mathrm{d} \boldsymbol{s}^{\mathrm{d}}\right]}_{\mathrm{d}\left(\frac{1}{2} \mathrm{~s}^{\mathrm{d}} \otimes \boldsymbol{s}^{\mathrm{d}}\right)}+\frac{\partial \tilde{\phi}^{\mathrm{d}}}{\partial q^{\mathrm{d}}} \mathrm{d} q^{\mathrm{d}} \\
& =\mathrm{d} \phi^{\mathrm{d}}=\frac{\partial \phi^{\mathrm{d}}}{\partial\left(\frac{1}{2} \boldsymbol{s}^{\mathrm{d}} \otimes \boldsymbol{s}^{\mathrm{d}}\right)}: \mathrm{d}\left(\frac{1}{2} \boldsymbol{s}^{\mathrm{d}} \otimes \boldsymbol{s}^{\mathrm{d}}\right)+\frac{\partial \phi^{\mathrm{d}}}{\partial q^{\mathrm{d}}} \mathrm{d} q^{\mathrm{d}} \\
& =\frac{\partial \phi^{\mathrm{d}}}{\partial\left(\frac{1}{2} \boldsymbol{s}^{\mathrm{d}} \otimes \boldsymbol{s}^{\mathrm{d}}\right)} \boldsymbol{s}^{\mathrm{d}} \cdot \mathrm{d} \boldsymbol{s}^{\mathrm{d}}+\frac{\partial \phi^{\mathrm{d}}}{\partial q^{\mathrm{d}}} \mathrm{d} q^{\mathrm{d}}
\end{aligned}
$$

implying

$$
\boldsymbol{n}_{\tilde{\phi}^{\mathrm{d}}}=\frac{\partial \phi^{\mathrm{d}}}{\partial\left(\frac{1}{2} \boldsymbol{s}^{\mathrm{d}} \otimes \boldsymbol{s}^{\mathrm{d}}\right)} \boldsymbol{s}^{\mathrm{d}}=\boldsymbol{s}^{\mathrm{d}} \frac{\partial \phi^{\mathrm{d}}}{\partial\left(\frac{1}{2} \boldsymbol{s}^{\mathrm{d}} \otimes \boldsymbol{s}^{\mathrm{d}}\right)},
$$

and $\partial \phi^{\mathrm{d}} / \partial q=\partial \tilde{\phi}^{\mathrm{d}} / \partial q$. Hence, the evolution formulas (3.13) and (3.22) define the same (maximum) damage dissipation along the direction of the current stress $\boldsymbol{s}^{\mathrm{d}}$. For future use and in general, we write the damage evolution equation

$$
\dot{\mathbf{d}}^{\mathrm{d}}=\gamma^{\mathrm{d}} \boldsymbol{N}_{\phi}^{\mathrm{d}}
$$

with $N_{\phi^{\mathrm{d}}}$ given by Eq. (3.13) or Eq. (3.22). The general relation

$$
\boldsymbol{n}_{\tilde{\phi}^{\mathrm{d}}}=\boldsymbol{N}_{\phi^{\mathrm{d}}} \boldsymbol{s}^{\mathrm{d}}=\boldsymbol{s}^{\mathrm{d}} \boldsymbol{N}_{\phi^{\mathrm{d}}}
$$

is also satisfied in both cases by Eq. (3.25). Given this relation, we can express the rate of the damage strains as

$$
\dot{\boldsymbol{e}}^{\mathrm{d}}=\mathbf{d}^{\mathrm{d}} \dot{\boldsymbol{s}}^{\mathrm{d}}+\dot{\mathbf{d}}^{\mathrm{d}} \boldsymbol{s}^{\mathrm{d}}=\mathbf{d}^{\mathrm{d}} \dot{\boldsymbol{s}}^{\mathrm{d}}+\gamma^{\mathrm{d}} \boldsymbol{n}_{\tilde{\phi}^{\mathrm{d}}}
$$

or, equivalently,

$$
\dot{\boldsymbol{s}}^{\mathrm{d}}=\mathbf{c}^{\mathrm{d}}\left(\dot{\boldsymbol{e}}^{\mathrm{d}}-\gamma^{\mathrm{d}} \boldsymbol{n}_{\tilde{\phi}^{\mathrm{d}}}\right)
$$

involving the current secant damage tensor $\mathbf{c}^{\mathrm{d}}=\mathbf{d}^{\mathrm{d}^{-1}}$. We note that the "degrading strains" (rates), referred to in Section 1, correspond to the last term in Eq. (3.28). We conclude then that it is the first term on the right-hand side of Eq. (3.28), which is also active in unloading, which gives the recoverable character of the damage strains $\boldsymbol{e}^{\mathrm{d}}$.

The damage tangent associated to this generic quadratic damage mechanism is also obtained using the Eqs. (2.51) and (2.55), leading to the expressions

(i) Damaged unloading:

$$
\mathbf{c}_{l u}^{\mathrm{d}}=\mathbf{c}^{\mathrm{d}}
$$


(ii) Loading (persistent damage):

$$
\mathbf{c}_{l u}^{\mathrm{d}}=\mathbf{c}^{\mathrm{d}}-\frac{1}{\Delta^{\mathrm{d}}} \mathbf{c}^{\mathrm{d}} \boldsymbol{n}_{\tilde{\phi}^{\mathrm{d}}} \otimes \mathbf{c}^{\mathrm{d}} \boldsymbol{n}_{\tilde{\phi}^{\mathrm{d}}},
$$

for

$$
\Delta^{\mathrm{d}}=\boldsymbol{n}_{\tilde{\phi}^{\mathrm{d}}}: \mathbf{c}^{\mathrm{d}} \boldsymbol{n}_{\tilde{\phi}^{\mathrm{d}}}+\underbrace{K^{\mathrm{d}}\left(\frac{\partial \tilde{\phi}^{\mathrm{d}}}{\partial q^{\mathrm{d}}}\right)^{2}}_{\tilde{\Delta}^{\mathrm{d}}},
$$

$$
\text { where } K^{\mathrm{d}}:=\frac{\mathrm{d}^{2} \mathscr{H}^{\mathrm{d}}}{\mathrm{d} \alpha^{\mathrm{d}^{2}}},
$$

the hardening/softening tangent modulus. We note the nature of $\tilde{\Delta}^{\mathrm{d}}$ as discussed in Section 3.1.1. The final global tangent is obtained using the general formula (2.69). We note again that the damage space basis $\mathbb{P}^{\mathrm{d}}$ is to be defined below for the cases of interest.

Fig. 2 sketches the linear response in unloading and reloading observed in a uniaxial tension test for the quadratic damage potential considered herein when combined with a quadratic elastic potential. The reduced one-dimensional setting provided by this simple stress of state requires only the consideration of the elastic stiffness characterized by the Young modulus $E^{\mathrm{e}}$ and the damage compliance contribution characterized by the scalar $D^{\mathrm{d}}=E^{\mathrm{d}^{-1}}$. We can observe again in this figure the recoverable character of the damage strain and the corresponding potential $W^{\mathrm{d}}(\cdot)$. Similarly, the apparent axial stiffness of the material upon unloading (corresponding to the global tangent (2.69) for this case) can be obtained from this figure as $E=E^{\mathrm{d}} E^{\mathrm{e}} /\left(E^{\mathrm{d}}+E^{\mathrm{e}}\right)$, showing the damage introduced by the considered formulation.

\section{Remark 3.2.}

1. Similar to the arguments presented in Remark 3.1 , the constraint on the damage dissipation $\mathscr{D}^{\mathrm{d}} \geqslant 0$ is automatically enforced by starting directly from a damage surface of the form

$$
\tilde{\phi}^{\mathrm{d}}\left(\boldsymbol{s}^{\mathrm{d}}, q^{\mathrm{d}}\right)=\tilde{g}^{\mathrm{d}}\left(\boldsymbol{s}^{\mathrm{d}}\right)-(\underbrace{\tilde{\omega}_{0}^{\mathrm{d}}-q^{\mathrm{d}}\left(\alpha^{\mathrm{d}}\right)}_{\tilde{\omega}^{\mathrm{d}}\left(\alpha^{\mathrm{d}}\right) \geqslant 0}) \leqslant 0
$$

for a positively homogeneous function $\tilde{g}^{\mathrm{d}}(\cdot)$ of degree $m$, so that

$\frac{\partial \tilde{g}^{\mathrm{d}}}{\partial \boldsymbol{s}^{\mathrm{d}}} \cdot \boldsymbol{s}^{\mathrm{d}}=\boldsymbol{n}_{\tilde{\phi}^{\mathrm{d}}} \cdot \boldsymbol{s}^{\mathrm{d}}=m \tilde{\boldsymbol{g}}^{\mathrm{d}}\left(\boldsymbol{s}^{\mathrm{d}}\right)$.

Combining Eq. (3.11) with Eq. (3.22), we obtain after some straightforward algebraic manipulations

$$
\mathscr{D}^{\mathrm{d}}=\frac{m}{2} \gamma^{\mathrm{d}}\left[\tilde{\omega}_{0}^{\mathrm{d}}-\left(1-\frac{2}{m}\right) q^{\mathrm{d}}\right] \text {. }
$$

Therefore, we conclude $\mathscr{D}^{\mathrm{d}} \geqslant 0$ for a homogeneous function of degree $m=2$. For the case of a homogeneous function of degree $m=1$, a positive dissipation is also concluded if $q^{\mathrm{d}} \geqslant-\tilde{\omega}_{0}^{\mathrm{d}}$; in particular, for the case of a softening law $q^{\mathrm{d}} \geqslant 0$.

2. Regarding the formula (3.22) for the evolution of the damage compliance $\boldsymbol{d}^{\mathrm{d}}$, we note that the denominator is nonzero; in fact

$\boldsymbol{n}_{\tilde{\phi}^{\mathrm{d}}} \cdot \boldsymbol{s}^{\mathrm{d}}>0$ for $\tilde{\phi}^{\mathrm{d}}\left(\boldsymbol{s}^{\mathrm{d}}, q^{\mathrm{d}}\right)=0$ and $\boldsymbol{s}^{\mathrm{d}} \neq 0$,

if $\tilde{\phi}^{\mathrm{d}}$ defines a convex elastic damage domain in the space of damage stresses $\boldsymbol{s}^{\mathrm{d}}\left(q^{\mathrm{d}}=\mathrm{constant}\right)$ including the origin $s^{\mathrm{d}}=0$, as it is usually the case. In fact, for the common case (3.33) with $\tilde{g}^{\mathrm{d}}(\cdot)$ homogeneous of degree $m>0$, we have 
$\boldsymbol{n}_{\tilde{\phi}^{\mathrm{d}}} \cdot \boldsymbol{s}^{\mathrm{d}}=m \tilde{\boldsymbol{g}}^{\mathrm{d}}\left(\boldsymbol{s}^{\mathrm{d}}\right)=m \tilde{\omega}^{\mathrm{d}}\left(\alpha^{\mathrm{d}}\right)>0$

as long as $0 \leqslant \tilde{\omega}^{\mathrm{d}}\left(\alpha^{\mathrm{d}}\right) \neq 0$, i.e., when the elastic domain does not reduce to $\boldsymbol{s}^{\mathrm{d}} \equiv 0$ (or, in other words, when it has not shrunk to the origin due to a softening of the material). In this situation, the relation (3.22) reflects the physical fact $\boldsymbol{d}^{\mathrm{d}} \rightarrow \infty$ for a fully damaged material; note that $\mathbf{c}^{\mathrm{d}} \rightarrow 0$ as needed in the expressions (2.51) and (2.55) of the tangent.

\subsection{Some existing models}

The generic quadratic model developed in Section 3.1 is fully defined once the damage surfaces (3.23), defining in the process the damage space (2.4) and the nature of the damage compliance (3.2), are defined. With specific particular definitions of these surfaces, the considered general formulation encompasses the models developed in Ortiz (1985) and Simo and Ju (1987a,b), and later considered in Hansen and Schreyer (1994), Simo et al. (1993) and Govindjee et al. (1995) in the modeling of the damage in brittle materials, concrete in particular. We show in the next sections that other existing stress-based damage models can be obtained in this way. We consider for simplicity the case of a linear elastic material, i.e., with a quadratic stored energy function

$$
W^{\mathrm{e}}\left(\boldsymbol{\varepsilon}^{\mathrm{e}}\right)=\frac{1}{2} \boldsymbol{\varepsilon}^{\mathrm{e}}: \mathbf{C}^{\mathrm{e}} \boldsymbol{\varepsilon}^{\mathrm{e}}
$$

for constant elasticities $\mathbf{C}^{\mathrm{e}}$, leading to the quadratic complementary energy function

$$
\chi^{\mathrm{e}}(\boldsymbol{\sigma})=\frac{1}{2} \boldsymbol{\sigma}: \mathbf{D}^{\mathrm{e}} \boldsymbol{\sigma}=\frac{1}{2}(\boldsymbol{\sigma} \otimes \boldsymbol{\sigma}): \mathbf{D}^{\mathrm{e}}
$$

for $\mathbf{D}^{\mathrm{e}}=\mathbf{C}^{\mathrm{e}^{-1}}$.

\subsubsection{An effective stress anisotropic damage model}

The formulation of anisotropic damage models can be often found in the literature developed through the introduction of the so-called damage tensors defining an effective stress tensor. Early examples are the works of Cordebois and Sidoroff (1982) and Murakami (1983). In the context developed in Section 3.2, we first note the secant relation

$$
\boldsymbol{\varepsilon}-\boldsymbol{\varepsilon}^{\mathrm{p}}=\boldsymbol{\varepsilon}^{\mathrm{e}}+\boldsymbol{\varepsilon}^{\mathrm{d}}=\left[\mathbf{D}^{\mathrm{e}}+\mathbf{D}^{\mathrm{d}}\right] \boldsymbol{\sigma}=\mathbf{D}^{\mathrm{e}}\left[\llbracket+\mathbf{C}^{\mathrm{e}} \mathbf{D}^{\mathrm{d}}\right] \boldsymbol{\sigma}
$$

for the fourth-order identity tensor $\square$. Hence, we can write

$$
\begin{aligned}
& \overline{\boldsymbol{\sigma}}:=\mathbf{C}^{\mathrm{e}}\left(\boldsymbol{\varepsilon}-\boldsymbol{\varepsilon}^{\mathrm{p}}\right)=\boldsymbol{M}^{-1} \boldsymbol{\sigma} \\
& \text { for } \boldsymbol{M}:=\left[\mathbb{q}+\mathbf{C}^{\mathrm{e}} \mathbf{D}^{\mathrm{d}}\right]^{-1},
\end{aligned}
$$

and the effective stresses $\overline{\boldsymbol{\sigma}}$. Numerous anisotropic damage models have been formulated through the consideration of a general effective stress tensor $\boldsymbol{M}$; we refer to Lemaitre and Chaboche (1978, 1985), Cordebois and Sidoroff (1982) and Murakami (1983) among others.

It is instructive to formulate the resulting anisotropic effective stress model in its classical form, without the explicit consideration of the damage strains $\boldsymbol{\varepsilon}^{\mathrm{d}}$. We first note the relations

$$
\boldsymbol{\sigma}=\mathbf{C}^{\mathrm{e}} \boldsymbol{\varepsilon}^{\mathrm{e}}=\mathbf{D}^{\mathrm{d}^{-1}} \boldsymbol{\varepsilon}^{\mathrm{d}} \Rightarrow \boldsymbol{\sigma}=\mathbf{C}(\underbrace{\boldsymbol{\varepsilon}-\varepsilon^{\mathrm{p}}}_{\varepsilon^{\mathrm{e}}+\varepsilon^{\mathrm{d}}}) \quad \text { for } \mathbf{C}:=\boldsymbol{M} \mathbf{C}^{\mathrm{e}}
$$

and with $\boldsymbol{M}$ defined in Eq. (3.41b). This last expression relates directly the stress with the reversible part of the strain $\varepsilon-\varepsilon^{p}$ through the current (degraded) material tangent $\mathbf{C}$. The thermodynamic framework presented in Section 2 can then be written equivalently, after some straightforward algebraic manipulations, as 


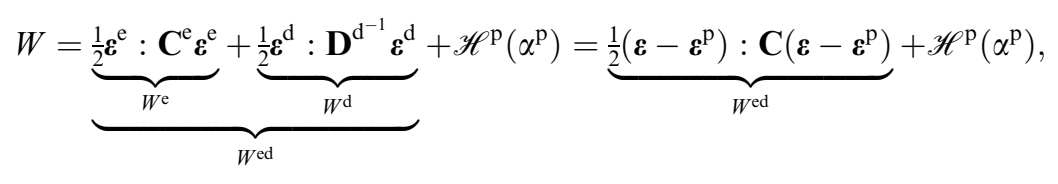

in terms of the total $\varepsilon$ and plastic $\varepsilon^{\mathrm{p}}$ strains, as it is used extensively in the literature. In fact, this expression of the total stored energy in combination with the second term of the stress/strain relation (3.42) leads to the following expression of the total dissipation

$$
\mathscr{D}:=\boldsymbol{\sigma}: \dot{\boldsymbol{\varepsilon}}-\dot{W}=\underbrace{\boldsymbol{\sigma}: \dot{\boldsymbol{\varepsilon}}^{\mathrm{p}}+\mathbf{q}^{\mathrm{p}} \dot{\alpha}^{\mathrm{p}}}_{\mathscr{P}^{\mathrm{p}}}+\underbrace{\frac{1}{2} \boldsymbol{\sigma}: \dot{\mathbf{D}} \boldsymbol{\sigma}}_{\mathscr{D}^{\mathrm{d}}} \text { for } \mathbf{D}:=\mathbf{C}^{-1}
$$

without the explicit consideration of the damage strains. The natural decomposition of the dissipation in a plastic and a damage component, as it was the case for the framework considered herein (Eq. (2.10)), becomes apparent.

We observe that the framework developed in this work identifies directly an evolution for the effective damage tensor $\boldsymbol{M}$. Namely, using the evolution equation (3.26), we obtain

$$
\dot{\boldsymbol{M}}=-\gamma^{\mathrm{d}} \boldsymbol{M} \mathbf{C}^{\mathrm{e}} \boldsymbol{N}_{\phi^{\mathrm{d}}} \boldsymbol{M}
$$

for $\boldsymbol{N}_{\phi^{\mathrm{d}}}$ given by Eq. (3.13) or Eq. (3.22) for a general damage function $\phi^{\mathrm{d}}\left(\frac{1}{2}(\boldsymbol{\sigma} \otimes \boldsymbol{\sigma}), q^{\mathrm{d}}\right) \leqslant 0$ or $\tilde{\phi}^{\mathrm{d}}\left(\boldsymbol{\sigma}, q^{\mathrm{d}}\right) \leqslant 0$, respectively. More importantly, this evolution equation is obtained in the thermodynamic framework developed in Section 2 and, in particular, through the direct physical modeling of the damage mechanism through the stress/damage strain relations characterizing it. This situation is to be contrasted with usual ad-hoc characterizations of the evolution of the effective stress tensor commonly found in the literature.

Remark 3.3. Having defined the effective stresses $\overline{\boldsymbol{\sigma}}$ in terms of the damage tensor $\boldsymbol{M}$ in Eq. (3.41), one may consider the yield criterion Eq. (2.25b) in the effective stress space, that is,

$$
\bar{\phi}^{\mathrm{p}}\left(\overline{\boldsymbol{\sigma}}, q^{\mathrm{p}}\right) \leqslant 0
$$

as it is commonly found in the literature (Hansen and Schreyer, 1994; Ju, 1989). This option, however, leads to a nonsymmetric tangent.

\subsubsection{An isotropic damage model}

Damage models in the form of a scalar damage variable characterizing the isotropic damage of the material can be traced to the pioneering work of Kachanov (1958). Isotropic damage can be characterized by the consideration damage surface

$$
\phi^{\mathrm{d}}\left(\boldsymbol{\sigma}, q^{\mathrm{d}}\right)=\chi^{\mathrm{e}}(\boldsymbol{\sigma})-\left(\omega_{0}^{\mathrm{d}}-q\right) \leqslant 0
$$

in terms of the elastic complementary energy function (3.39). An equivalent expression (using twice the square root of $\chi^{\mathrm{e}}$ ) can be found in Simo and Ju (1987a,b). The threshold value to activate the damage is denoted by $\omega_{0}^{\mathrm{d}}$ and evolves with the scalar hardening/softening variable $q$, given in terms of the conjugate strain-like variable $\alpha^{\mathrm{d}} \in[0, \infty)$ by Eq. (3.17) for the hardening/softening potential $\mathscr{H}^{\mathrm{d}}\left(\alpha^{\mathrm{d}}\right)$. The appearance of all the components of the stress $\boldsymbol{\sigma}$ (not specific components $\boldsymbol{\sigma}^{\mathrm{d}}=\mathbb{P}^{\mathrm{d}^{\mathrm{T}}} \boldsymbol{s}^{\mathrm{d}}$ only) in the damage surface (3.47) identifies the associated damage space with the full space of symmetric tensors, that is,

$$
\mathscr{V}^{\mathrm{d}} \equiv \mathscr{S} .
$$


In this situation, we can choose $\boldsymbol{e}^{\mathrm{d}} \equiv \boldsymbol{\varepsilon}^{\mathrm{d}}$ (and $\boldsymbol{s}^{\mathrm{d}} \equiv \boldsymbol{\sigma}^{\mathrm{d}}=\boldsymbol{\sigma}$ ), with no need to introduce the projections $\left\{\mathbb{P}_{a}^{\mathrm{d}}\right\}_{a=1}^{n_{\mathrm{d}}}$ in Eq. (2.6) (or, in other words, they are the identity). Similarly, we work with the global damage compliance $\mathbf{D}^{\mathrm{d}} \equiv \mathbf{d}^{\mathrm{d}}$ in this case.

The damage evolution equation (3.13) read in this case

$$
\begin{aligned}
& \dot{\mathbf{D}}^{\mathrm{d}}=\gamma^{\mathrm{d}} \mathbf{D}^{\mathrm{e}}, \\
& \dot{\alpha}^{\mathrm{d}}=\gamma^{\mathrm{d}}
\end{aligned}
$$

for the elastic compliance $\mathbf{D}^{\mathrm{e}}$. For the particular case (3.39) involving a constant $\mathbf{D}^{\mathrm{e}}$, the damage evolution equations (3.49) and (3.50) can be easily integrated to arrive at the expression

$$
\mathbf{D}^{\mathrm{d}}=\int \dot{\mathbf{D}} \mathrm{d} t=\int \dot{\alpha}^{\mathrm{d}} \mathrm{d} t \mathbf{D}^{\mathrm{e}}=\alpha^{\mathrm{d}} \mathbf{D}^{\mathrm{e}}
$$

The damage strains are easily obtained using Eq. (2.40) with the complementary function (3.6), leading to

$$
\boldsymbol{\varepsilon}^{\mathrm{d}}=\mathbf{D}^{\mathrm{d}} \boldsymbol{\sigma}=\alpha^{\mathrm{d}} \mathbf{D}^{\mathrm{e}} \boldsymbol{\sigma}=\alpha^{\mathrm{d}} \boldsymbol{\varepsilon}^{\mathrm{e}},
$$

thus resulting in the relations

$$
\begin{aligned}
& \boldsymbol{\varepsilon}^{\mathrm{e}}=\frac{1}{\left(1+\alpha^{\mathrm{d}}\right)}\left(\boldsymbol{\varepsilon}-\boldsymbol{\varepsilon}^{\mathrm{p}}\right)=(1-d)\left(\boldsymbol{\varepsilon}-\boldsymbol{\varepsilon}^{\mathrm{p}}\right), \\
& \boldsymbol{\varepsilon}^{\mathrm{d}}=\frac{\alpha^{\mathrm{d}}}{1+\alpha^{\mathrm{d}}}\left(\boldsymbol{\varepsilon}-\boldsymbol{\varepsilon}^{\mathrm{p}}\right)=d\left(\boldsymbol{\varepsilon}-\boldsymbol{\varepsilon}^{\mathrm{p}}\right)
\end{aligned}
$$

after introducing the definition

$$
d:=\frac{\alpha^{\mathrm{d}}}{1+\alpha^{\mathrm{d}}} \Rightarrow d \in[0,1]
$$

In this notation, we can write

$$
\mathbf{D}^{\mathrm{d}}=\frac{d}{1-d} \mathbf{D}^{\mathrm{e}}
$$

for the damage secant compliance $\mathbf{D}^{\mathrm{d}}$. The stress is given by

$$
\boldsymbol{\sigma}=\mathbf{C}^{\mathrm{e}} \boldsymbol{\varepsilon}^{\mathrm{e}}=(1-d) \mathbf{C}^{\mathrm{e}}\left(\boldsymbol{\varepsilon}-\boldsymbol{\varepsilon}^{\mathrm{p}}\right)=(1-d) \overline{\boldsymbol{\sigma}} \text { for } \overline{\boldsymbol{\sigma}}:=\mathbf{C}^{\mathrm{e}}\left(\boldsymbol{\varepsilon}-\boldsymbol{\varepsilon}^{\mathrm{p}}\right)
$$

with $\mathbf{C}^{\mathrm{e}}=\mathbf{D}^{\mathrm{e}^{-1}}$. Relations (3.53) and (3.57) define the classical concepts of effective strains and stresses, respectively, in the isotropic case, as it can be found in the classical literature on the subject (see the complete account in Kachanov (1986)). Eq. (3.57) is a particular case of the general anisotropic relation (3.41), with $\boldsymbol{M}=(1-d)$. In fact, the elastic potential (3.43) reads in this case

$$
W=(1-d) \frac{1}{2}\left(\boldsymbol{\varepsilon}-\boldsymbol{\varepsilon}^{\mathrm{p}}\right): \mathbf{C}^{\mathrm{e}}\left(\boldsymbol{\varepsilon}-\boldsymbol{\varepsilon}^{\mathrm{p}}\right)+\mathscr{H}^{\mathrm{p}}\left(\alpha^{\mathrm{p}}\right)
$$

with the coupled plastic damage dissipation expressed equivalently as

$$
\mathscr{D}:=\boldsymbol{\sigma}: \dot{\boldsymbol{\varepsilon}}-\dot{W}=\underbrace{\boldsymbol{\sigma}: \dot{\boldsymbol{\varepsilon}}^{\mathrm{p}}+\boldsymbol{q}^{\mathrm{p}} \dot{\alpha}^{\mathrm{p}}}_{\mathscr{P}^{\mathrm{p}}}+\underbrace{\dot{d} Y}_{\mathscr{D}^{\mathrm{d}}} \text { for } Y:=\frac{1}{2}\left(\boldsymbol{\varepsilon}-\boldsymbol{\varepsilon}^{\mathrm{p}}\right): \mathbf{C}^{\mathrm{e}}\left(\boldsymbol{\varepsilon}-\boldsymbol{\varepsilon}^{\mathrm{p}}\right),
$$

two expressions without an explicit reference to the damage strains $\boldsymbol{\varepsilon}^{\mathrm{d}}$, as found extensively in the literature. 
The damage tangent moduli $\mathbf{C}_{l u}^{\mathrm{d}}$ given by Eqs. (3.30) and (3.31) reads in this case

(i) Damaged unloading:

$\mathbf{C}_{l u}^{\mathrm{d}}=\frac{(1-d)}{d} \mathbf{C}^{\mathrm{e}}$

(ii) Damage loading:

$$
\mathbf{C}_{l u}^{\mathrm{d}}=\frac{(1-d)}{d}\left[\mathbf{C}^{\mathrm{e}}-\frac{1}{\overline{\boldsymbol{\sigma}}: \mathbf{D}^{\mathrm{e}} \overline{\boldsymbol{\sigma}}+\frac{K^{\mathrm{d} d} d}{(1-d)^{3}}} \overline{\boldsymbol{\sigma}} \otimes \overline{\boldsymbol{\sigma}}\right] .
$$

Finally, the total tangent moduli

$$
\dot{\boldsymbol{\sigma}}=\mathbf{C}^{\mathrm{epd}} \dot{\boldsymbol{\varepsilon}}
$$

given by the general formula (2.69), reads in this case of a single isotropic damage mechanism

(i) Damaged unloading:

$\mathbf{C}^{\mathrm{epd}}=(1-d) \mathbf{C}^{\mathrm{ep}}$.

(ii) Damage loading:

$$
\mathbf{C}^{\mathrm{epd}}=(1-d)\left[\mathbf{C}^{\mathrm{ep}}-\frac{1}{\overline{\boldsymbol{\sigma}}: \mathbf{D}^{\mathrm{e}} \overline{\boldsymbol{\sigma}}+\frac{K^{\mathrm{d} d}}{(1-d)^{4}}} \overline{\boldsymbol{\sigma}} \otimes \overline{\boldsymbol{\sigma}}\right]
$$

for the isotropic hardening/softening modulus $K^{\text {d }}$ defined in Eq. (3.32b). The case of elastic damage is recovered when $\mathbf{C}^{\mathrm{ep}}=\mathbf{C}^{\mathrm{e}}$.

\subsubsection{Smeared crack models and strong discontinuities}

The modeling of cracking in concrete has been often formulated in the framework of the so-called smeared crack models, especially in the context of finite element analyses. Representative early applications of this approach can be found in Rashid (1968), Bazant and Cedolin (1979) and Rots et al. (1985) among many others. Despite its popularity, the lack of a sound thermodynamic framework as opposed to continuum damage models has, perhaps, made this approach less attractive from the theoretical point of view. We show in Section 3.2.3 that smeared crack models fit perfectly in the thermodynamic framework of continuum damage models encompassed by the simple generic quadratic model presented above.

For a given nucleation criterion (say Rankine's maximum stress criterion for brittle materials), a single crack can be characterized by its normal direction, the unit vector $\boldsymbol{n}$. The cohesive opening/closing of the crack is then assumed controlled by the traction vector associate to $\boldsymbol{n}$, that is,

$$
\boldsymbol{T}=\boldsymbol{\sigma n} .
$$

Let $\left\{\boldsymbol{m}_{1} \equiv \boldsymbol{n}, \ldots, \boldsymbol{m}_{n_{\mathrm{dim}}}\right\}$ denote an orthonormal Cartesian system in a general $n_{\mathrm{dim}}$ dimensional setting (so $\boldsymbol{m}_{\mathrm{i}} \cdot \mathbf{m}_{j}=\delta_{i j}$ for $\left.i, j=1, n_{\mathrm{dim}}\right)$. Denoting the corresponding components of the traction vector $\boldsymbol{T}$ by

$$
s_{a}^{\mathrm{d}} \equiv T_{a}:=\boldsymbol{m}_{a} \cdot \boldsymbol{T} \text { for } a=1, n_{\mathrm{dim}},
$$

the general damage surface (3.23) in terms of the reduced damage stresses $\boldsymbol{s}^{\mathrm{d}}$ corresponds in this case to a damage surface in terms of the traction vector on the crack. These considerations identify the damage space 
$\mathscr{V}^{\mathrm{d}}$ for the damage mechanism associated to a single crack. Namely, the projection matrices into this space are given by

$$
\mathbb{P}_{a}^{\mathrm{d}}=\frac{1}{2}\left(\boldsymbol{m}_{a} \otimes \boldsymbol{n}+\boldsymbol{n} \otimes \boldsymbol{m}_{a}\right) \quad \text { for } a=1, n_{\mathrm{dim}}
$$

(so $\mathbb{P}_{1}^{\mathrm{d}}=\boldsymbol{n} \otimes \boldsymbol{n}$, in particular). The orthogonality relation (2.5) can be easily verified. The damage strains are then constructed by Eq. (2.4) in terms of the so-called crack strains $\boldsymbol{e}^{\mathrm{d}}$, with $n_{\mathrm{d}}=n_{\mathrm{dim}}$ in this case.

The damage evolution equation (3.26) reads in this case

$$
\dot{\mathbf{d}}^{\mathrm{d}}=\gamma^{\mathrm{d}} \boldsymbol{N}_{\phi^{\mathrm{d}}},
$$

for the reduced crack compliance $\mathbf{d}^{\mathrm{d}} \in \mathbb{R}^{n_{\text {dim }} \times n_{\text {dim }}}$, a rank two (not four) tensor in this case, with $N_{\phi^{\mathrm{d}}}$ given by Eq. (3.13) or Eq. (3.22), in terms of a damage surface involving the components of the traction vector $\boldsymbol{T}$. We refer to Kroplin and Weihe (1997), among many others, for the determination of such damage surfaces for the modeling of cracking in concrete.

The inviscid smeared crack models considered in this section in combination of strain softening are known to lead to fundamental difficulties, the well-known pathological mesh dependence of the resulting finite element solutions in particular. To avoid these inconsistencies, we have presented in Armero (1997a,b) an alternative formulation of this type of anisotropic damage models in the context of the so-called strong discontinuities. This approach considers the limit solutions with a discontinuous displacement field (Simo et al., 1993; Armero and Garikipati, 1996). More recently, we have presented in Armero (1997a, 1999) a multi-scale framework for the introduction of these solutions, or better the corresponding localized dissipative mechanisms, in the large scale problem of the local continuum. The corresponding strains in the small scale (a local neighborhood of a point $\boldsymbol{x}$ ) are given

$$
\boldsymbol{\varepsilon}_{\mu}=\overline{\boldsymbol{\varepsilon}}_{\mu}+(\boldsymbol{\xi} \otimes \boldsymbol{n})^{s} \delta_{\Gamma_{x}},
$$

for the Dirac delta function $\delta_{\Gamma_{x}}$ associated to the discontinuity surface $\Gamma_{x}$ with unit normal $\boldsymbol{n}$, with a regular part of the local strain $\overline{\boldsymbol{\varepsilon}}_{\mu}$ (determining the stress $\boldsymbol{\sigma}$ ) and a jump displacement field $\boldsymbol{\xi}$ across $\Gamma_{x}$. The decomposition (3.69) fits along the developments presented in this section with $\boldsymbol{\varepsilon}^{\mathrm{e}} \equiv \overline{\boldsymbol{\varepsilon}}_{\mu}$ and $\boldsymbol{\varepsilon}^{\mathrm{d}}=$ $\mathbb{P}^{\mathrm{d}^{\mathrm{T}}} \boldsymbol{e}^{\mathrm{d}}$ with $\mathbb{P}_{a}^{\mathrm{d}}$ defined as in Eq. (3.68) and $e_{a}^{\mathrm{d}}=\xi_{a} \delta_{\Gamma_{x}}\left(a=1, n_{\mathrm{dim}}\right)$.

The damage potential $W^{\mathrm{d}}(\cdot)$ is given in this case by

$$
\widehat{W}^{\mathrm{d}}\left(\boldsymbol{e}^{\mathrm{d}} ; \mathscr{I}^{\mathrm{d}}\right)=\widetilde{W}^{\mathrm{d}}\left(\boldsymbol{\xi} ; \widetilde{\mathscr{I}}^{\mathrm{d}}\right) \delta_{\Gamma_{x}},
$$

for a set of localized internal variables $\mathscr{I}^{\mathrm{d}}=\widetilde{\mathscr{I}}^{\mathrm{d}} \delta_{\Gamma_{x}}$. Taking the differential of Eq. (3.70), we obtain

$$
\mathrm{d} \widehat{W}^{\mathrm{d}}=\frac{\partial \widetilde{W}^{\mathrm{d}}}{\partial \boldsymbol{\xi}} \cdot \mathrm{d}(\underbrace{\xi \delta_{\Gamma_{x}}}_{\boldsymbol{e}^{\mathrm{d}}})+\frac{\partial \widetilde{W}^{\mathrm{d}}}{\partial \widetilde{\mathscr{I}}^{\mathrm{d}}} \odot \mathrm{d}(\underbrace{\widetilde{\mathscr{I}}^{\mathrm{d}} \delta_{\Gamma_{x}}}_{\mathscr{I}^{\mathrm{d}}}) \Rightarrow\left\{\begin{array}{l}
\boldsymbol{s}^{\mathrm{d}}=\frac{\partial \widetilde{W}^{\mathrm{d}}}{\partial \xi}, \\
\mathscr{Q}^{\mathrm{d}}=-\frac{\partial \widetilde{W}^{\mathrm{d}}}{\partial \widetilde{\mathscr{I}}^{\mathrm{d}}},
\end{array}\right.
$$

being both regular distributions. For the quadratic model (3.1), the relation (3.70) leads to $\mathbf{d}^{\mathrm{d}}=\widetilde{\mathbf{d}}^{\mathrm{d}} \delta_{\Gamma_{x}}$ and $\alpha^{\mathrm{d}}=\widetilde{\alpha}^{\mathrm{d}} \delta_{\Gamma_{x}}$, with the secant relation (3.8) and the constitutive relation (3.10) reading in this case

$$
\begin{aligned}
& \xi=\widetilde{\mathbf{d}}^{\mathrm{d}} \boldsymbol{s}^{\mathrm{d}} \\
& q^{\mathrm{d}}=-\frac{\partial \widetilde{\mathscr{H}^{\mathrm{d}}}}{\partial \widetilde{\alpha}^{\mathrm{d}}}
\end{aligned}
$$

for a localized softening potential $\widetilde{\mathscr{H}}^{\mathrm{d}}\left(\widetilde{\alpha}^{\mathrm{d}}\right)$. 
The dissipation (3.11) is then given by

$$
\mathscr{D}^{\mathrm{d}}=[\underbrace{\frac{1}{2}\left(\boldsymbol{s}^{\mathrm{d}} \otimes \boldsymbol{s}^{\mathrm{d}}\right): \dot{\tilde{\mathbf{d}}}^{\mathrm{d}}+q^{\mathrm{d}} \dot{\widetilde{\alpha}}^{\mathrm{d}}}_{\widetilde{\mathscr{D}}^{\mathrm{d}}}] \delta_{\Gamma_{x}},
$$

reflecting its localized nature. The damage evolution equation (3.26) read now

$$
\dot{\tilde{\mathbf{d}}}^{\mathrm{d}}=\widetilde{\gamma}^{\mathrm{d}} \boldsymbol{N}_{\phi^{\mathrm{d}}} \quad \text { and } \quad \dot{\tilde{\alpha}}^{\mathrm{d}}=\widetilde{\gamma}^{\mathrm{d}} \frac{\partial \phi^{\mathrm{d}}}{\partial q^{\mathrm{d}}},
$$

for $\boldsymbol{N}_{\phi^{\mathrm{d}}}$ defined as in Eq. (3.13) or Eq. (3.22), and a localized damage consistency parameter localized on $\Gamma_{x}$ (with $\gamma^{\mathrm{d}}=\widetilde{\gamma}^{\mathrm{d}} \delta_{\Gamma_{x}}$ ), satisfying similarly the Kuhn-Tucker loading-unloading conditions (2.27) and consistency conditions (2.28). The damage surfaces $\phi^{\mathrm{d}}$ are defined as considered above for smeared crack models, but now resulting directly in the stress-displacement (3.72a) relations, with associated compliance $\widetilde{\mathbf{d}}^{\mathrm{d}}$. These relations are directly introduced in the finite element solutions through a local enhancement of the finite elements; we refer to the aforementioned references for complete details.

\section{Concluding remarks}

We have presented in this first paper a general framework for the formulation of continuum damage models in the infinitesimal range. This framework is based on the kinematic assumption of the strains decomposed in elastic, plastic and damage parts, similar to the elastoplastic decompositions of the strains in plasticity models. The inclusion in this assumption of a general thermodynamic framework describing the response of the material leads then to a complete characterization of the damage in the material. Most notably, these considerations allow for a complete and independent characterization of the damage mechanisms in contrast with more traditional approaches found in the literature based on the effective stress concept through the evolution of the so-called "damage tensors". The resulting formulation leads then to a very modular treatment of the damage effects in solids, requiring only the physically motivated modeling of the strain/stress response associated to the damage mechanism independently of the overall response of the solid. In addition, we have also shown that classical models, from isotropic damage models to smeared crack models, fit in the proposed formulation, thus giving a must needed unified framework to these existing approaches.

In conclusion, this work has identified a framework leading to a physically motivated modeling of plastic damage in materials with an efficient numerical treatment. Even though the concept of damage strain used herein can be found in the literature as noted in the introduction, most notably through the so-called "crack strains" in the modeling of damage in concrete, their use in the formulation of continuum damage models seem not to be widely spread. We believe that the highly modular structure of the proposed formulation clearly justifies its consideration, even in the case when the formulation reduces to existing models. This is especially the case when developing numerical schemes for the integration of the resulting constitutive models as we present in Part II of this work in combination with a simple plastic damage model of porous metals. We intend to continue this work with the consideration of additional couplings between the different inelastic mechanisms (see Remark 3.3) as well as the extension of these ideas to the finite deformation range. 


\section{Acknowledgements}

Financial support for this research has been provided by the ONR under contract No. N00014-96-10818 with UC Berkeley. This support is gratefully acknowledged. We also acknowledge the support of the NSF under contract No. CMS-9703000 with UC Berkeley.

\section{References}

Armero, F., 1997a. On the characterization of localized solutions in inelastic solids: an analysis of wave propagation in a softening bar. Comp. Meth. Appl. Mech. Engng., available also as UCB/SEMM Report no. 97/18, submitted for publication.

Armero, F., 1997b. Localized anisotropic damage of brittle materials. In: Owen, D.R.J., Onate, E., Hinton, E. (Eds.), Proc. COMPLASV, CIMNE, Barcelona.

Armero, F., 1999. Large-scale modeling of localized dissipative mechanisms in a local continuum: applications to the numerical simulation of strain localization in rate-dependent inelastic solids. Mech. Cohesive-Frict. Mater. 4, 101-131.

Armero, F., Garikipati, K., 1996. An analysis of strong discontinuities in multiplicative finite strain plasticity and their relation with the numerical simulation of strain localization in solids. Int. J. Solids Struct. 33, 2863-2885.

Armero, F., Li, S., 1998. Numerical simulation of anisotropic damage at finite strains. Proc. 12th ASCE Engng. Mech. Conf., La Jolla, CA.

Armero, F., Oller, S., 1999. A general framework for continuum damage models part II: applications to the numerical simulation of porous metals. Int. J. Solids Struct. 37, 7437-7464.

Bazant, Cedolin, 1979. Blunt crack band propagation in finite element analysis. J. Engng. Mech. Div. ASCE 105, 297-315.

Bazant, Z., Oh, J., 1983. Crack band theory for fracture of concrete. Mater. Struct. RILEM 16, 155-177.

Bazant, Z., Oh, B.H., 1985. Microplane model for progressive fracture of concrete and rock. J. Engng. Mech. 111, 559-582.

Carol, I., Rizzi, E., Willam, K., 1994. Unified theory of elastic degradation and damage based on a loading surface. Int. J. Solids Struct. 31, 2835-2865.

Chaboche, J.L., 1995. A continuum damage theory with anisotropic and unilateral damage. La Recherche Aerospatiale 2, $139-147$.

Cordebois, J., Sidoroff, F., 1982. Endommagement anisotrope. J. Mecanique Theor. Appl., special.

Dougill, J.W., 1976. On stable progressively fracturing solids. ZAMP 27, 423-437.

Golub, G.H., Loan, C.F., 1989. Matrix Computations, second ed. The John Hopkins University Press, Baltimore.

Govindjee, S.D, Kay, G.J., Simo, J.C., 1995. Anisotropic modeling and numerical simulation of brittle damage in concrete. Int. J. Num. Meth. Engng. 38, 3611-3633.

Hansen, N.R., Schreyer, H.L., 1994. A thermodynamically consistent framework for theories of elastoplasticity coupled with damage. Int. J. Solids Struct. 31, 359-389.

Hansen, N.R., Schreyer, H.L., 1995. Damage deactivation. ASME J. Appl. Mech. 62, 450-458.

Hueckel, T., Maier, G., 1977. Incrementally boundary-value problems in the presence of coupling of elastic and plastic deformations: a rock mechanics oriented theory. Int. J. Solids Struct. 13, 1-15.

Ju, J.W., 1989. On energy-based coupled elastoplastic damage theories: constitutive modeling and computation aspects. Int. J. Solids Struct. 25, 803-833.

Kachanov, L.M., 1958. Time rupture process under creep conditions. Izv. ARad. SSSR Teckh. Nauk 8, 26-31 in Russian.

Kachanov, L.M., 1986. Introduction to Continuum Damage Mechanics. Martinus Nijhoff Publishers, Dordrecht.

Kroplin, B., Weihe, S., 1997. Constitutive and Geometrical Aspects of Fracture Induced Anisotropy. In: Owen, D.R.J., Onate, E., and Hinton, E., (Eds.), Proc. COMPLAS V, CIMNE, Barcelona.

Lemaitre, J., Chaboche, J.L., 1978. Aspect phénoménologique de la rupture per endommagement. J. Mécanique Appliquée 2, $317-365$.

Lemaitre, J., Chaboche, J.L., 1985. Mécanique de Matériaux Solide, Dunod, Paris.

Lubliner, 1990. Plasticity Theory. MacMillan, New York.

Lubliner, J., Oliver, J., Oller, S., Onate, E., 1989. A plastic-damage model for concrete. Int. J. Solids Struct. 25, $299-326$.

Luccioni, B., Oller, S., Danesi, R., 1996. Coupled plastic-damage model. Comp. Meth. Appl. Mech. Engng. 129, 81-89.

Murakami, S., 1983. Notion of continuum damage mechanics and its application to anisotropic creep damage theory. J. Engng. Mater. Technol. 105, 99-105.

Ortiz, M., 1985. A constitutive theory for the inelastic behavior of concrete. Mech. Mater. 4, 67-93.

Ortiz, M., 1987. Analytical study of the localized failure modes of concrete. Mech. Mater. 6, 159-174.

Rashid, Y.R., 1968. Analysis of prestressed concrete pressure vessels. Nucl. Engng. Design 7, 334-344.

Rots, J.G., Nauta, P., Kusters, G., Blaauwendraa, T., 1985. Smeared Crack Approach and Fracture Localization in Concrete. Heron 30

Simo, J.C., Hughes, T.J.R., 1998. Computational Inelasticity. Springer, Verlag. 
Simo, J.C., Ju, J.W., 1987a. Strain- and stress-based continuum damage models Part I: formulation. Int. J. Solids Struct. $23,821-840$. Simo, J.C., Ju, J.W., 1987b. Strain- and Stress-Based Continuum Damage Models Part II: Computational Aspects. Int. J. Solids Struct. 23, 841-869.

Simo, J.C., Oliver, J., Armero, F., 1993. An analysis of strong discontinuities induced by softening solutions in rate independent solids. J. Comput. Mech. 12, 277-296.

Truesdell, Noll 1965. The Nonlinear Field Theories of Mechanics. Handbich der Physik Bd. III/3 In: Fluegge, S., Springer, Berlin. Yazadani, S., Schreyer, H.L., 1988. An anisotropic damage model with dilatation for concrete. Mech. Mater. 7, $231-244$. 